\title{
Development and analysis of the Soil Water Infiltration Global database
}

Mehdi Rahmati ${ }^{1,2}$, Lutz Weihermüller ${ }^{2,3}$, Jan Vanderborght ${ }^{2,3}$, Yakov A. Pachepsky ${ }^{4}$, Lili Mao ${ }^{5}$, Seyed Hamidreza Sadeghi ${ }^{6}$, Niloofar Moosavi ${ }^{2}$, Hossein Kheirfam ${ }^{7}$, Carsten Montzka ${ }^{2,3}$, Kris Van Looy ${ }^{2,3}$, Brigitta Toth ${ }^{8,94}$, Zeinab Hazbavi ${ }^{6}$, Wafa Al Yamani ${ }^{9}$, Ammar A. Albalasmeh ${ }^{10}$, Ma'in Z. Alghzawi ${ }^{10}$, Rafael Angulo-Jaramillo ${ }^{11}$, Antônio Celso Dantas Antonino ${ }^{12}$,

George Arampatzis ${ }^{13}$, Robson André Armindo ${ }^{14}$, Hossein Asadi ${ }^{15}$, Yazidhi Bamutaze ${ }^{16}$, Jordi Batlle-Aguilar ${ }^{17,18,19}$, Béatrice Béchet ${ }^{20}$, Fabian Becker ${ }^{21}$, Günter Blöschl ${ }^{22,23}$, Klaus Bohne ${ }^{24}$, Isabelle Braud $^{25}$, Clara Castellano ${ }^{26}$, Artemi Cerdà ${ }^{27}$, Maha Chalhoub $^{17}$, Rogerio Cichota $^{28}$, Milena Císlerováa $^{29}$, Brent Clothier ${ }^{30}$, Yves Coquet ${ }^{17,31}$, Wim Cornelis ${ }^{32}$, Corrado Corradini ${ }^{33}$, Artur Paiva Coutinho ${ }^{12}$, Muriel Bastista de Oliveira ${ }^{34}$, José Ronaldo de Macedo ${ }^{35}$, Matheus Fonseca Durães ${ }^{14}$, Hojat Emami ${ }^{36}$, Iraj Eskandari ${ }^{37}$, Asghar Farajnia ${ }^{38}$, Alessia Flammini ${ }^{33}$, Nándor Fodor ${ }^{39}$, Mamoun Gharaibeh ${ }^{10}$, Mohamad Hossein Ghavimipanah ${ }^{6}$, Teamrat A. Ghezzehei ${ }^{40}$, Simone Giertz ${ }^{41}$, Evangelos G. Hatzigiannakis ${ }^{13}$, Rainer Horn ${ }^{42}$, Juan José Jiménez ${ }^{43}$, Diederik Jacques $^{44}$, Saskia Deborah Keesstra ${ }^{45,46}$, Hamid Kelishadi ${ }^{47}$, Mahboobeh Kiani-Harchegani ${ }^{6}$, Mehdi Kouselou ${ }^{1}$, Madan Kumar Jha ${ }^{48}$, Laurent Lassabatere ${ }^{11}$, Xiaoyan $\mathbf{L i}^{49}$, Mark A. Liebig ${ }^{50}$, Lubomír Lichner $^{51}$, María Victoria López ${ }^{52}$, Deepesh Machiwal ${ }^{53}$, Dirk Mallants ${ }^{54}$, Micael Stolben Mallmann ${ }^{55}$, Jean Dalmo de Oliveira Marques ${ }^{56}$, Miles R. Marshall ${ }^{57}$, Jan Mertens ${ }^{58}$, Félicien Meunier ${ }^{59}$, Mohammad Hossein Mohammadi ${ }^{15}$, Binayak P. Mohanty ${ }^{60}$,

Mansonia Pulido-Moncada ${ }^{61}$, Suzana Montenegro ${ }^{62}$, Renato Morbidelli ${ }^{33}$, David Moret-Fernández ${ }^{52}$, Ali Akbar Moosavi ${ }^{63}$, Mohammad Reza Mosaddeghi ${ }^{47}$, Seyed Bahman Mousavi ${ }^{1}$, Hasan Mozaffari ${ }^{63}$, Kamal Nabiollahi ${ }^{64}$, Mohammad Reza Neyshabouri ${ }^{65}$, Marta Vasconcelos Ottoni ${ }^{66}$,

Theophilo Benedicto Ottoni Filho ${ }^{67}$, Mohammad Reza Pahlavan-Rad ${ }^{68}$, Andreas Panagopoulos ${ }^{13}$, Stephan Peth ${ }^{69}$, Pierre-Emmanuel Peyneau ${ }^{20}$, Tommaso Picciafuoco ${ }^{22,33}$, Jean Poesen ${ }^{70}$,

Manuel Pulido ${ }^{71}$, Dalvan José Reinert ${ }^{72}$, Sabine Reinsch ${ }^{57}$, Meisam Rezaei ${ }^{32,93}$, Francis Parry Roberts $^{57}$, David Robinson $^{57}$, Jesús Rodrigo-Comino ${ }^{73,74}$, Otto Corrêa Rotunno Filho ${ }^{75}$, Tadaomi Saito ${ }^{76}$, Hideki Suganumaa $^{77}$, Carla Saltalippi $^{33}$, Renáta Sándor ${ }^{39}$, Brigitta Schütt $^{21}$, Manuel Seeger ${ }^{74}$, Nasrollah Sepehrnia ${ }^{78}$, Ehsan Sharifi Moghaddam ${ }^{6}$, Manoj Shukla ${ }^{79}$, Shiraki Shutaro ${ }^{80}$, Ricardo Sorando ${ }^{25}$, Ajayi Asishana Stanley ${ }^{81}$, Peter Strauss ${ }^{82}$, Zhongbo Su ${ }^{83}$, Ruhollah Taghizadeh-Mehrjardi $^{84}$, Encarnación Taguas ${ }^{85}$, Wenceslau Geraldes Teixeira ${ }^{86}$, Ali Reza Vaezi ${ }^{87}$, Mehdi Vafakhah ${ }^{6}$, Tomas Vogel ${ }^{29}$, Iris Vogeler ${ }^{28}$, Jana Votrubova ${ }^{29}$, Steffen Werner ${ }^{88}$, Thierry Winarski ${ }^{11}$, Deniz Yilmaz ${ }^{89}$, Michael H. Young ${ }^{90}$, Steffen Zacharias ${ }^{91}$, Yijian Zeng ${ }^{83}$, Ying Zhao ${ }^{92}$, Hong Zhao ${ }^{83}$, and Harry Vereecken ${ }^{2,3}$

${ }^{1}$ Department of Soil Science and Engineering, Faculty of Agriculture, University of Maragheh, Maragheh, Iran

${ }^{2}$ Forschungszentrum Jülich GmbH, Institute of Bio- and Geosciences: Agrosphere (IBG-3), Jülich, Germany

${ }^{3}$ ISMC International Soil Modeling Consortium, Institute of Bio and Geosciences Forschungszentrum Jülich, 52425 Jülich, Germany

${ }^{4}$ USDA-ARS Environmental Microbial and Food Safety Laboratory, Beltsville, MD 20705, USA

${ }^{5}$ Key Laboratory of Dryland Agriculture, Ministry of Agriculture, Institute of Environment and Sustainable Development in Agriculture, Chinese Academy of Agricultural Sciences, Beijing 100081, PR China

${ }^{6}$ Department of Watershed Management Engineering, Faculty of Natural Resources, Tarbiat Modares University, Iran

${ }^{7}$ Department of Environmental Sciences, Urmia Lake Research Institute, Urmia University, Urmia, Iran 
${ }^{8}$ Institute for Soil Sciences and Agricultural Chemistry, Centre for Agricultural Research, Hungarian Academy of Sciences, Budapest, Hungary

${ }^{9}$ Environment Agency, Abu Dhabi, UAE

${ }^{10}$ Department of Natural Resources and Environment, Faculty of Agriculture, Jordan University of Science and Technology, P.O. Box 3030, Irbid 22110, Jordan

${ }^{11}$ Univ Lyon, Université Claude Bernard Lyon 1, CNRS, ENTPE, UMR5023 LEHNA, 69518, Vaulx-en-Velin, France

${ }^{12}$ Universidade Federal de Pernambuco, Centro Acadêmico do Agreste, Núcleo de Tecnologia, Caruaru, Brazil

${ }^{13}$ Hellenic Agricultural Organization, Soil and Water Resources Institute, 57400 Sindos, Greece

${ }^{14}$ Department of Physics (DFI), Federal University of Lavras (UFLA), P.O. Box 3037, CEP 37200-000, Lavras, Brazil

${ }^{15}$ Department of Soil Science, Faculty of Agricultural Engineering and Technology, University of Tehran, Karaj, Iran

${ }^{16}$ Department of Geography, Geo-Informatics and Climatic Sciences, Makerere University, P.O. Box 7062, Kampala, Uganda

${ }^{17}$ UMR 1402 INRA AgroParisTech Functional Ecology and Ecotoxicology of Agroecosystems, Institut National de la Recherche Agronomique, AgroParisTech B.P. 01, 78850 Thiverval-Grignon, France

${ }^{18}$ UMR 8148 IDES CNRS/Université Paris-Sud, XI Bât. 504, Faculté des Sciences 91405, Orsay CEDEX, France

${ }^{19}$ Innovative Groundwater Solutions (IGS), Victor Harbor, 5211, South Australia, Australia ${ }^{20}$ IFSTTAR, GERS, EE, 44344 Bouguenais, France

${ }^{21}$ Freie Universität Berlin, Department of Earth Sciences, Institute of Geographical Sciences, Malteserstr. 74-100, Lankwitz, 12249, Berlin, Germany

${ }^{22}$ Centre for Water Resource Systems, TU Wien, Karlsplatz 13, 1040 Vienna, Austria

${ }^{23}$ Institute of Hydraulic Engineering and Water Resources Management, TU Wien, Karlsplatz 13/222, 1040 Vienna, Austria

${ }^{24}$ Faculty of Agricultural and Environmental Sciences, University of Rostock, Germany

${ }^{25}$ Irstea, UE RiverLy, Lyon-Villeurbanne Center, 69625 Villeurbanne, France

${ }^{26}$ Pyrenean Institute of Ecology-CSIC, AV. Montañana 1005,

Av. Victoria s/n. 50059 Zaragoza, 22700 Jaca, Huesca, Spain

${ }^{27}$ Soil Erosion and Degradation Research Group, Department of Geography, University of Valencia, Valencia, Spain

${ }^{28}$ Plant and Food Research, Mount Albert Research Station, Auckland, New Zealand

${ }^{29}$ Czech Technical University in Prague, Faculty of Civil Engineering,

Thákurova 7, 16629 Prague 6, Czech Republic

${ }^{30}$ Plant and Food Research, Palmerston North, New Zealand

${ }^{31}$ ISTO UMR 7327 Université d'Orléans, CNRS, BRGM, 45071 Orléans, France

${ }^{32}$ Department of Soil Management, UNESCO Chair on Eremology, Ghent University, Ghent, Belgium

${ }^{33}$ Department of Civil and Environmental Engineering, University of Perugia, Perugia, Italy

${ }^{34}$ UniRedentor University Center. BR 356, 25, Presidente Costa e Silva, Itaperuna, Rio de Janeiro, Brazil

${ }^{35}$ Embrapa Solos, Rua Jardim Botânico, 1.024, CEP 22040-060, Jardim Botânico, Rio de Janeiro, RJ, Brazil

${ }^{36}$ Department of Soil Science, Faculty of Agriculture, Ferdowsi University of Mashhad, Mashhad, Iran

${ }^{37}$ Dryland Agricultural Research Institute, Agricultural Research, Education and Extension

Organization Maragheh, East Azerbaijan, Iran

${ }^{38}$ Scientific Member of Soil and Water Research Department, East Azerbaijan Agricultural and

Natural Resources Research and Education center, Iran

${ }^{39}$ Agricultural Institute, Centre for Agricultural Research, Hungarian Academy of Sciences, Brunszvik str. 2., 2462 Martonvásár, Hungary

${ }^{40}$ Life and Environmental Sciences, University of California, Merced, USA

${ }^{41}$ Geographisches Institut, Universität Bonn, Bonn, Germany

${ }^{42}$ Institute of Plant Nutrition and Soil Science, Christian-Albrechts-Universität zu Kiel, Olshausenstr. 40, 24118 Kiel, Germany

${ }^{43}$ ARAID Researcher, Instituto Pirenaico de Ecología, Spanish National Research Council (IPE-CSIC), Avda. Llano de la victoria 16, Jaca (Huesca), 22700, Spain 
${ }^{44}$ Enginereed and Geosystems Analysis Unit, Belgian Nuclear Research Centre, Mol, Belgium

${ }^{45}$ Soil, Water and Land Use Team, Wageningen Environmental Research, Wageningen UR, 6708PB Wageningen, the Netherlands

${ }^{46}$ Civil, Surveying and Environmental Engineering,

the University of Newcastle, Callaghan 2308, Australia

${ }^{47}$ Department of Soil Science, College of Agriculture, Isfahan University of Technology, Isfahan 84156-83111, Iran

${ }^{48}$ Agricultural and Food Engineering Department, Indian Institute of Technology Kharagpur, Kharagpur - 721302, West Bengal, India

${ }^{49}$ State Key Laboratory of Earth Surface Processes and Resource Ecology,

Faculty of Geographical Science, Beijing Normal University, Beijing 100875, China

${ }^{50}$ Research Soil Scientist, USDA Agricultural Research Service, Mandan, ND, USA

${ }^{51}$ Institute of Hydrology, Slovak Academy of Sciences, Bratislava, Slovakia

${ }^{52}$ Departamento de Suelo y Agua, Estación Experimental de Aula Dei (EEAD), Consejo Superior de Investigaciones Científicas (CSIC), P.O. Box 13034, 50080 Zaragoza, Spain

${ }^{53}$ ICAR-Central Arid Zone Research Institute, Regional Research Station, Kukma - 370105, Bhuj, Gujarat, India

${ }^{54}$ CSIRO Land and Water, Glen Osmond, South Australia, Australia

${ }^{55}$ Soil Science Graduate Program (ufsm.br/ppgcs), Federal University of Santa Maria, state of Rio Grande do Sul, Brazil

${ }^{56}$ Federal Institute of Education, Science and Technology of the Amazonas - IFAM, Campus Center of Manaus, Manaus, Brazil

${ }^{57}$ Centre for Ecology \& Hydrology, Environment Centre Wales, Deiniol Road, Bangor, Gwynedd LL57 2UW, UK

${ }^{58}$ ENGIE Research and Technologies, Simon Bolivardlaan 34, 1000 Brussels, Belgium

${ }^{59}$ Université catholique de Louvain, Earth and Life Institute-Environmental Sciences, Louvain-la Neuve, Belgium

${ }^{60}$ Department of Biological and Agricultural Engineering, 2117 TAMU, Texas A\&M Univ., College Station, TX 77843-2117, USA

${ }^{61}$ Aarhus University, Department of Agroecology, Research Centre Foulum, Blichers Allé 20, P.O. Box 50, 8830 Tjele, Denmark

${ }^{62}$ Universidade Federal de Pernambuco (UFPE), Av. Prof. Moraes Rego, 1235-Cidade Universitária, Recife-PE-CEP: 50670-901, Brazil

${ }^{63}$ Department of Soil Science, College of Agriculture, Shiraz University, Shiraz, Iran

${ }^{64}$ Department of Soil Science and Engineering, Faculty of Agriculture, University of Kurdistan, Sanandaj, Kurdistan Province, Iran

${ }^{65}$ Department of Soil Science, Faculty of Agriculture, University of Tabriz, Tabriz, Iran ${ }^{66}$ Department of Hydrology, Geological Survey of Brazil (CPRM), Av. Pauster, 404. CEP 22290-240, Rio de Janeiro, Brazil

${ }^{67}$ Department of Water Resources and Environment, Federal University of Rio de Janeiro, Avenida Athos da Silveira Ramos, P.O. Box 68570, Rio de Janeiro, RJ, Brazil

${ }^{68}$ Soil and Water Research Department, Sistan Agricultural and Natural Resources Research and Education Center, Agricultural Research, Education and Extension Organization (AREEO), Zabol, Iran ${ }^{69}$ Department of Soil Science, University of Kassel, Nordbahnhofstr. 1a, 37213 Witzenhausen, Germany

${ }^{70}$ Department of Earth and Environmental Sciences, Catholic University of Leuven, Geo-Institute, Celestijnenlaan 200E, 3001 Heverlee, Belgium

${ }^{71}$ GeoEnvironmental Research Group, University of Extremadura, Faculty of Philosophy and Letters, Avda. de la Universidad s/n, 10071 Cáceres, Spain

${ }^{72}$ Soil Science Department, Federal University of Santa Maria, state of Rio Grande do Sul, Brazil

${ }^{73}$ Instituto de Geomorfología y Suelos, Department of Geography, University of Málaga, 29071, Málaga, Spain

${ }^{74}$ Department of Physical Geography, Trier University, 54286 Trier, Germany

${ }^{75}$ Civil Engineering Program, Alberto Luiz Coimbra Institute for Postgraduate Studies and Research in Engineering (COPPE), Federal University of Rio de Janeiro, Avenida Athos da Silveira Ramos, Rio de Janeiro, RJ, Brazil 


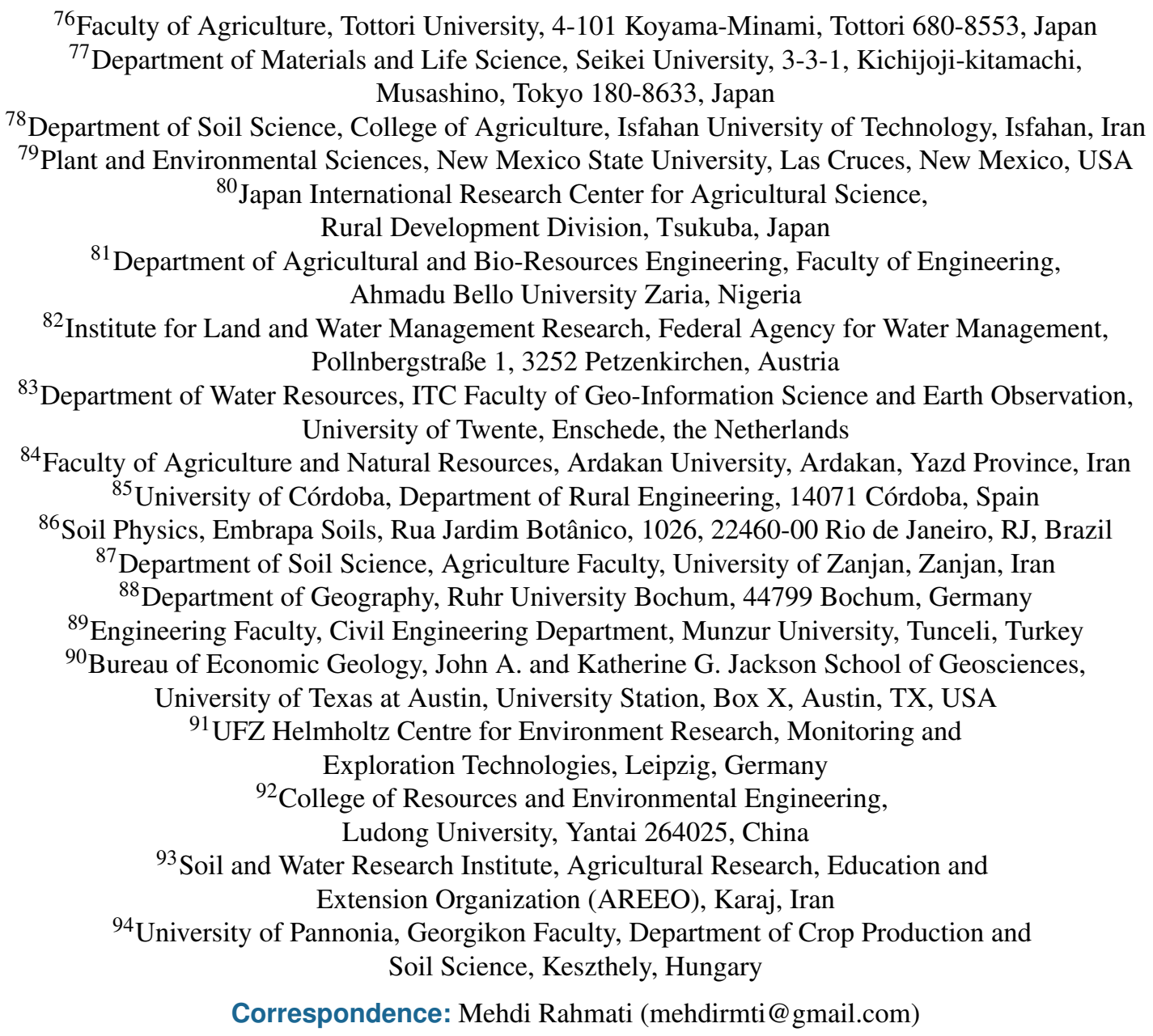

Received: 28 January 2018 - Discussion started: 6 March 2018

Revised: 12 June 2018 - Accepted: 13 June 2018 - Published: 10 July 2018

Abstract. In this paper, we present and analyze a novel global database of soil infiltration measurements, the Soil Water Infiltration Global (SWIG) database. In total, 5023 infiltration curves were collected across all continents in the SWIG database. These data were either provided and quality checked by the scientists who performed the experiments or they were digitized from published articles. Data from 54 different countries were included in the database with major contributions from Iran, China, and the USA. In addition to its extensive geographical coverage, the collected infiltration curves cover research from 1976 to late 2017. Basic information on measurement location and method, soil properties, and land use was gathered along with the infiltration data, making the database valuable for the development of pedotransfer functions (PTFs) for estimating soil hydraulic properties, for the evaluation of infiltration measurement methods, and for developing and validating infiltration models. Soil textural information (clay, silt, and sand content) is available for 3842 out of 5023 infiltration measurements $(\sim 76 \%)$ covering nearly all soil USDA textural classes except for the sandy clay and silt classes. Information on land use is available for $76 \%$ of the experimental sites with agricultural land use as the dominant type $(\sim 40 \%)$. We are convinced that the SWIG database will allow for a better parameterization of the infiltration process in land surface models and for testing infiltration models. All collected data and related soil characteristics are provided online in *.xlsx and *..ssv formats for reference, and we add a disclaimer that the database is for public domain use only and can be copied freely by referencing it. Supplementary data are available at https://doi.org/10.1594/PANGAEA.885492 (Rahmati et al., 2018). Data quality assessment is strongly advised prior to any use of this database. Finally, we would like to encourage scientists to extend and update the SWIG database by uploading new data to it. 


\section{Introduction}

Infiltration is the process by which water enters the soil surface and it is one of the key fluxes in the hydrological cycle and the soil water balance. Water infiltration and the subsequent redistribution of water in the subsurface are two important processes that affect the soil water balance (Campbell, 1985; Hillel, 2013; Lal and Shukla, 2004; Morbidelli et al., 2011) and influence several soil processes and functions including availability of water and nutrients for plants, microbial activity, erosion rates, chemical weathering, and soil thermal and gas exchange between the soil and the atmosphere (Campbell, 1985). Infiltration plays a definitive role in maintaining soil system functions and as it is a key process that controls several of the United Nations goals for sustainability (Keesstra et al., 2016). The generation of surface runoff, a key factor in controlling floods, is also directly related to the infiltration process. Water that cannot infiltrate in the soil becomes available for surface runoff. Two main mechanisms are responsible for the generation of excess water that produce overland flow: Dunne saturation excess and Hortonian infiltration excess (Sahoo et al., 2008). Dunne overland flow, or saturation excess, occurs when the soil profile is completely saturated and precipitation can no longer infiltrate into soil. The Dunne mechanism is more common to near-channel areas or is generated from partial areas of the hillslope where water tables are shallowest (Sahoo et al., 2008). On the other hand, Hortonian overland flow is characterized by rainfall intensities exceeding the infiltration rate of the soil. In other words, during a rainfall event, water infiltration at the soil surface and runoff are highly dependent on the boundary conditions, namely, the rainfall intensity and the soil hydraulic properties. If the rainfall intensity is less than the soil infiltrability, water will completely infiltrate into the soil without any runoff (Hillel, 2013). In this case, the infiltration rate align with the rainfall intensity. Otherwise, if the precipitation intensity exceeds the soil infiltration rate at a certain moment in time, excess water will be generated even if the soil profile is unsaturated. In this case water will pond on the soil surface and become available for surface runoff. If this occurs, the boundary condition at the soil surface undergoes a shift in the dominant flow process from one governed by capillary action to one governed by pressures of hydraulic head. Assuming that the water pressure heads remain constant at the soil surface, the infiltration rate is described by a decreasing function over time, tending towards the value of the hydraulic conductivity function for the water pressure head imposed at the soil surface (AnguloJaramillo et al., 2016; Chow et al., 1988). In the past decades, water infiltration tests, using either ponded or tension infiltrometers, have been developed to quantify the cumulative infiltration at the soil surface. In these cases, the 3-D axisymmetric water infiltration corresponds to an upper boundary defined by a constant water pressure head or a series of constant water pressure heads. The infiltration process is quantified by determining the amount of water which infiltrates, over time, from which the cumulative infiltration, $I(t),(\mathrm{L})$, and the infiltration rate, $i(t),\left(\mathrm{L} \mathrm{T}^{-1}\right)$ can be derived. $i(t)$ and $I(t)$ are related to each other by derivation (Campbell, 1985; Hillel, 2013; Lal and Shukla, 2004):

$i(t)=\frac{\mathrm{d} I(t)}{\mathrm{d} t}$.

As stated above, the infiltration rate $i(t)$ is expected to decrease to a plateau defined by the value of the hydraulic conductivity corresponding to the imposed water pressure head plus a term related to radial water infiltration (Angulo et al., 2016). In the case of large rings, the final infiltration rate approaches the value of the hydraulic conductivity corresponding to the imposed water pressure head (gravity flow). Consequently, if water ponding is imposed at the surface, $i(t)$ tends towards the saturated hydraulic conductivity. Infiltration into the soil is controlled by several factors including soil properties (e.g., texture, bulk density, initial water content), layering, slope, cover condition (vegetation, crust, and/or stone), rainfall pattern (Smith et al., 2002; Corradini et al., 2017), and time. As soil texture and soil surface conditions (e.g., cover) are independent of time at the scale of individual infiltration events, these characteristics can be assumed to be constant during the event. On the other hand, soil structure, especially at the soil surface, can rapidly change, for instance, due to tillage, grazing, or the destruction of soil aggregates by rain drop impact. In dry soils, initial infiltration rates are substantially higher than the saturated hydraulic conductivity of the surface layer due to capillary effects which control the sorptivity of the soil. However, as infiltration proceeds, the gradient between the pressure head at the soil surface and the pressure head below the wetting front reduces over time so that the infiltration rate finally reaches a constant value that approximates saturated hydraulic conductivity (Chow et al., 1988).

Infiltration measurements have been largely used to estimate soil saturated hydraulic conductivity. This soil property is a key factor to correctly describe all the components of the soil and land surface hydrological balance and is essential in the appropriate design of irrigation systems. Within the literature it is clear that extensive efforts have been made to estimate this property from basic soil properties using pedotransfer functions (PTFs). PTFs are knowledge-based rules or equations that relate simple soil properties to those properties of soil that are more difficult to obtain (Van Looy et al., 2017). Most of these efforts have been based on measurements made on samples of disturbed or undisturbed soil material. With this infiltration database, data are now made available that may contribute to better predicting the saturated soil hydraulic conductivity and demonstrate the effect 
of, for example, vegetation and land management on the parameters of interest.

The Richards (1931) equation, Eq. (2), written as a function of soil water content which is often referred to as the Fokker-Planck water diffusion equation, can be used to derive the closed-form expression of the infiltration rate in partially saturated soils.

$$
\frac{\partial \theta}{\partial t}=\frac{\partial}{\partial z}\left(D_{z}(\theta) \frac{\partial \theta}{\partial z}+K_{z}(\theta)\right)
$$

where $\theta$ is the volumetric soil water content $\left(\mathrm{L}^{3} \mathrm{~L}^{-3}\right) ; t$ is the time $(\mathrm{T}) ; z$ is the vertical depth position $(\mathrm{L}) ; K(\theta)$ is the soil hydraulic conductivity $\left(\mathrm{L} \mathrm{T}^{-1}\right)$; and $D(\theta)$ is soil water diffusivity $\left(\mathrm{L}^{2} \mathrm{~T}^{-1}\right)$, which is defined by Eq. (3) (Childs and Collis-George, 1950; Klute, 1952):

$D_{z}(\theta)=K_{z}(\theta) \frac{\partial h}{\partial \theta}$,

where $h$ is the matric potential in head units (L). The exact relationships between soil water content, soil matric potential, and soil hydraulic conductivity are necessary to solve the Richards equation. Several solutions of the Richards equation and many empirical, conceptual, semi-analytical, and physically based models - e.g., Green and Ampt (1911), Philip (1957), Smith and Parlange (1978), Haverkamp et al. (1994), and Corradini et al. (2017) - have been introduced to describe the infiltration process over time, even for preferential flows, e.g., Lassabatere et al. (2014). Furthermore, several direct or indirect experimental systems have been introduced to measure soil infiltration in the laboratory or in the field under different conditions (Gupta et al., 1994; McKenzie et al., 2002; Mao et al., 2008a). Data obtained from these systems can also be used to deduce soil saturated hydraulic conductivity directly.

Methods developed to measure and quantify water infiltration in soil are generally time-consuming and costly. Therefore, PTFs have been developed and applied by many researchers - e.g., Jemsi et al. (2013), Parchami-Araghi et al. (2013), Kashi et al. (2014), Sarmadian and TaghizadehMehrjardi (2014), and Rahmati (2017) - in order to easily parameterize infiltration models. However, these PTFs have been developed for specific regions, often limiting their applicability. As already mentioned, a large number of publications reporting soil infiltration data is available, but these data are dispersed in the literature and often difficult to access. Therefore, the aim of this data paper is to present and make available a collection of infiltration data digitized from available literature and from published or unpublished data provided directly by researchers around the world. These data are accompanied by metadata, which provide information about the location of the infiltration measurement, soil properties, and land management. Finally, we will provide some first results highlighting the suitability of the database for further research. The main article is also accompanied by a supplement providing more detailed information about the different methodologies to measure soil infiltration. This is added because many of readers are likely not well versed in soil infiltration and its limitations in measurement and modeling. For more detailed information on this, readers could refer to Smith et al. (2002), Corradini et al. (2017), and Hopmans et al. (2006).

\section{Method and materials}

\subsection{Data collection}

We collected infiltration measurements from different countries or regions by contacting the data owners or by extracting infiltration data from published literature (Fig. 1). To do this, a data request was sent to potential data owners through different forums and email exchanges. The flyer asked data owners to cooperate in the development of the Soil Water Infiltration Global (SWIG) database by providing infiltration data as well as metadata about experimental conditions (e.g., initial soil moisture content at the start of the experiment and method used), soil properties, land use, topography, geographical coordinates of the sites, and any other relevant information to interpret the data and to increase the value of the database. Infiltration data reported in the literature were digitized and included in the database together with additional information provided in these papers. The digitization approach is discussed in Sect. 2.2. In total, 5023 single infiltration curves were collected, of which 510 infiltration curves were digitized from 74 published papers (Table 1) and 4513 were provided by 68 different research teams (Table 2), being published or unpublished data. The references and correspondences for data supplied by direct communications with researchers are also reported in Table 2. Therefore, users may refer to these references for detailed information about the applied methods or procedures.

\subsection{Data digitization}

In order to digitize infiltration curves reported in the literature, screenshots of the relevant plots were taken, and figures were imported into the plot digitizer 2.6.8 (Huwaldt and Steinhorst, 2015). First, the origin of the axes and the highest $x$ and $y$ values were defined and the diagram plane was spanned. Then, all point values were picked out and an output table with the $x-y$ pairs (time vs. infiltration rate or cumulative infiltration) was generated and stored.

\subsection{Database structure}

The SWIG database is prepared in *.xlsx with a backup file in *.csv formats containing several datasets. Supplementary data are available at https://doi.org/10.1594/PANGAEA. 885492 (Rahmati et al., 2018) . The first dataset, named "I_cm", contains cumulative infiltration data in centimeter 

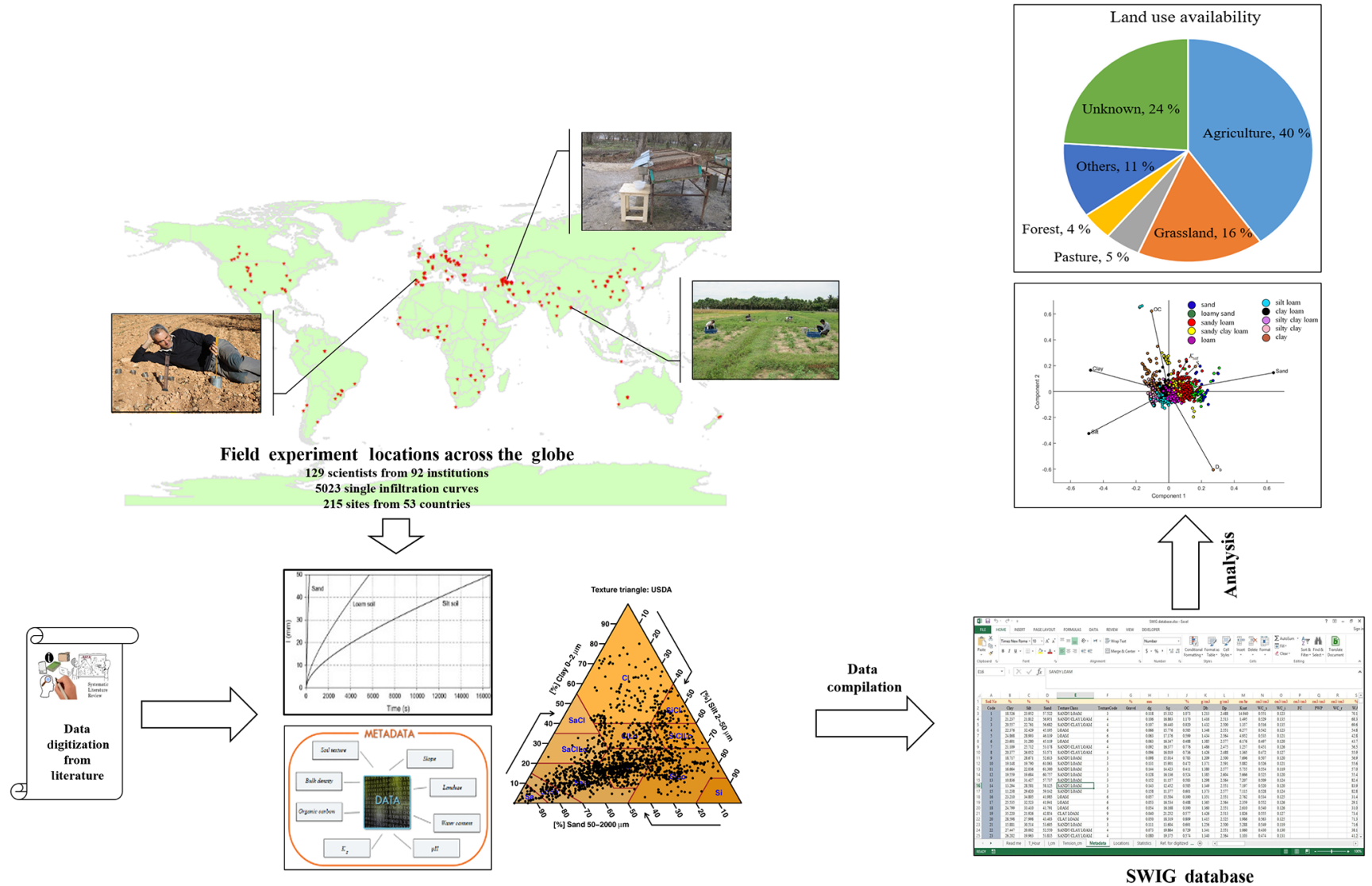

SWIG database

Figure 1. SWIG flowchart.

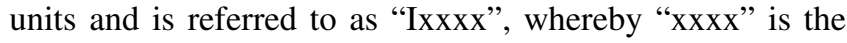
identifier of the individual infiltration test. The corresponding time intervals in hours for the infiltration data are labeled "T_Hour" and named "Txxxx". The constant or varying pressure or tension heads (if any) during infiltration measurements are also reported in another dataset named "Tension_cm". The database also contains additional variables and information relevant to the infiltration data provided by data owners or digitized from articles, as listed in Table 3, and which is labeled "Metadata". Additional soil properties were determined by different standards; therefore, data harmonization might be needed for some of those, especially in the case of water content at field capacity, $\mathrm{pH}$, or wet-aggregate stability. Further information on measurement methods is available from references of the data. Since the geometric mean diameter $\left(d_{\mathrm{g}}\right)$ and standard deviation $\left(S_{\mathrm{g}}\right)$ of soil particle sizes are rarely measured, both parameters were computed using the following equations (Shirazi and
Boersma, 1984):

$$
\begin{aligned}
& d_{\mathrm{g}}=\exp (a), \quad a=0.01 \sum_{i=1}^{n} f_{i} \ln D_{i}, \\
& S_{\mathrm{g}}=\exp (b), \quad b^{2}=0.01 \sum_{i=1}^{n} f_{i} \ln ^{2} D_{i}-a^{2},
\end{aligned}
$$

where $f_{i}$ is the percent of total soil mass having diameters equal to or less than the arithmetic mean of interval limits $\left(D_{i}\right)$ that define three main fractions $(i)$ of clay, silt, and sand with mean values of $0.001,0.026$, and $1.025 \mathrm{~mm}$, respectively. For the infiltration data, where the soil texture is unknown, $d_{\mathrm{g}}$ and $S_{\mathrm{g}}$ could not be calculated and the data field in the database was left empty. The database also contains the locations of the experimental sites in another dataset named "Locations" that provides the approximate latitude and longitude in decimal degree (dd.dd) format. Table 2 is also provided in the SWIG database in two other worksheets named "Ref. for digitized data" and "Ref. for data provided by owner". 
Table 1. References used to extract infiltration curves and metadata.

\begin{tabular}{|c|c|c|c|c|c|c|c|}
\hline \multirow{2}{*}{ Number } & \multicolumn{2}{|c|}{ Dataset } & \multirow{2}{*}{ Reference } & \multirow{2}{*}{ Number } & \multicolumn{2}{|c|}{ Dataset } & \multirow{2}{*}{ Reference } \\
\hline & From & To & & & From & To & \\
\hline 1 & 295 & 317 & Miller et al. (2005) & 38 & 4612 & - & Wang et al. (2016) \\
\hline 2 & 318 & 322 & Adindu Ruth et al. (2014) & 39 & 4613 & 4615 & Qian et al. (2014) \\
\hline 3 & 542 & 544 & Alagna et al. (2016) & 40 & 4617 & 4619 & Fan et al. (2013) \\
\hline 4 & 545 & - & Angulo-Jaramillo et al. (2000) & 41 & 4620 & - & Zhang et al. (2000) \\
\hline 5 & 546 & 548 & Su et al. (2016) & 42 & 4621 & 4623 & Wang et al. (2015a) \\
\hline 6 & 549 & 550 & Quadri et al. (1994) & 43 & 4624 & 4633 & Yang and Zhang (2011) \\
\hline 7 & 551 & 553 & Qi and Liu (2014) & 44 & 4634 & 4657 & Wu et al. (2016) \\
\hline 8 & 554 & 558 & Huang et al. (2015) & 45 & 4658 & 4663 & Ma et al. (2017) \\
\hline 9 & 559 & 568 & Al-Kayssi and Mustafa (2016) & 46 & 4664 & 4681 & Thierfelder et al. (2003) \\
\hline 10 & 1421 & 1432 & Bhardwaj and Singh (1992) & 47 & 4682 & 4683 & Commandeur et al. (1994) \\
\hline 11 & 1433 & 1435 & Berglund et al. (1980) & 48 & 4684 & 4686 & Di Prima et al. (2016) \\
\hline 12 & 1436 & 1443 & Wu et al. (2016) & 49 & 4687 & 4688 & Angulo-Jaramillo et al. (2000) \\
\hline 13 & 1444 & 1446 & Chartier et al. (2011) & 50 & 4689 & 4691 & Machiwal et al. (2006) \\
\hline 14 & 1447 & 1456 & Sihag et al. (2017) & 51 & 4692 & - & Ayu et al. (2013) \\
\hline 15 & 1457 & 1460 & Machiwal et al. (2006) & 52 & 4693 & 4699 & Rei et al. (2016) \\
\hline 16 & 1461 & 1466 & Igbadun et al. (2016) & 53 & 4700 & 4702 & Omuto et al. (2006) \\
\hline 17 & 1467 & 1469 & Mohanty et al. (1994) & 54 & 4703 & 4706 & Návar and Synnott (2000) \\
\hline 18 & 1470 & 1472 & Sauwa et al. (2013) & 55 & 4707 & - & Scotter et al. (1988) \\
\hline 19 & 1473 & 1476 & Arshad et al. (2015) & 56 & 4708 & 4720 & Khan and Strosser (1998) \\
\hline 20 & 1477 & 1488 & Bhawan (1997) & 57 & 4721 & 4724 & Lipiec et al. (2006) \\
\hline 21 & 1489 & 1495 & Uloma et al. (2013) & 58 & 4725 & - & Suzuki (2013) \\
\hline 22 & 1496 & - & Al-Azawi (1985) & 59 & 4726 & 4728 & Sukhanovskij et al. (2015) \\
\hline 23 & 1497 & 1499 & Ogbe et al. (2011) & 60 & 4729 & 4749 & Al-Ghazal (2002) \\
\hline 24 & 1500 & 1507 & Teague (2010) & 61 & 4750 & - & Sorman et al. (1995) \\
\hline 25 & 4506 & 4515 & Askari et al. (2008) & 62 & 4751 & 4764 & Bowyer-Bower (1993) \\
\hline 26 & 4516 & - & Delage et al. (2016) & 63 & 4765 & 4788 & Medinski et al. (2009) \\
\hline 27 & 4517 & 4518 & Ruprecht and Schofield (1993) & 64 & 4789 & 4792 & Latorre et al. (2015) \\
\hline 28 & 4519 & 4520 & Bertol et al. (2015) & 65 & 4793 & 4795 & Biro et al. (2010) \\
\hline 29 & 4521 & 4523 & Naeth et al. (1991) & 66 & 4796 & 4799 & Mohammed et al. (2007) \\
\hline 30 & 4524 & 4529 & Huang et al. (2011) & 67 & 4800 & 4815 & Abdallah et al. (2016) \\
\hline 31 & 4530 & 4537 & van der Kamp et al. (2003) & 68 & 4816 & 4819 & Murray and Buttle (2005) \\
\hline 32 & 4538 & - & Jačka et al. (2016) & 69 & 4820 & 4831 & Zhang et al. (2015) \\
\hline 33 & 4539 & 4568 & Matula (2003) & 70 & 4832 & 4837 & Perkins and McDaniel (2005) \\
\hline 34 & 4569 & 4586 & Casanova (1998) & 71 & 4838 & 4841 & Arriaga et al. (2010) \\
\hline 35 & 4587 & 4593 & Holzapfel et al. (1988) & 72 & 4842 & 4857 & Thierfelder et al. (2017) \\
\hline 36 & 4594 & 4605 & Wang et al. (2015b) & 73 & 4858 & 4867 & Thierfelder and Wall (2009) \\
\hline 37 & 4606 & 4611 & Mao et al. (2016) & 74 & 4868 & 4879 & Abagale et al. (2012) \\
\hline
\end{tabular}

\section{Results and discussion}

\subsection{Spatial and temporal data coverage}

The SWIG database (Rahmati et al., 2018) consists of 5023 soil water infiltration measurements spread over nearly all continents (Fig. 2). Data were derived from 54 countries (Table 4). The largest number of data sources were provided by scientists in Iran $(n=38)$, China $(n=23)$, and the USA $(n=15)$, whereby one data source might contain several water infiltration measurements. The SWIG database covers measurements from 1976 to 2017. A sparse coverage was obtained for the higher latitudes of the Northern Hemisphere (above $60^{\circ}$ ) including Norway, Finland, Sweden, Iceland,
Greenland, and Russia. The lack of reports with infiltration data from most countries of the former Soviet Union as well as the Sahelian and Saharan countries is also notable, as well as the small number of infiltration data from Australia. Figure 3 shows the number of samples by climatic zone (Rubel et al., 2017; Kottek et al., 2006). The majority of the data are from warm temperate, fully humid climate (49\%); arid steppe climate and warm temperate climate with dry summer are the second and third most represented climate zones with 22 and $12 \%$, respectively. Figures 4 and 5 show the frequency of experimental sites, respectively, by the World Reference Base (WRB) (IUSS, 2006) and USDA soil taxonomy systems (USDA, 2014) based on the SoilGrids dataset (Hengl et al., 2017). Regarding the WRB classification sys- 
Table 2. References and correspondence for data supplied by data owners.

\begin{tabular}{|c|c|c|c|c|c|}
\hline \multirow{2}{*}{ Number } & \multicolumn{2}{|c|}{ Dataset } & \multirow{2}{*}{ Contact person } & \multirow{2}{*}{ Email for contact } & \multirow{2}{*}{ Reference } \\
\hline & From & To & & & \\
\hline 1 & 1 & 135 & M. Rahmati & mehdirmti@gmail.com & Rahmati (2017) \\
\hline 2 & 136 & 294 & A. Farajnia & farajnia1966@yahoo.com & Unpublished data \\
\hline 3 & 323 & 376 & M. Shukla & shuklamk@nmsu.edu & Shukla et al. $(2003,2006)$ \\
\hline 4 & 377 & 426 & S. H. R. Sadeghi & sadeghi@modares.ac.ir & $\begin{array}{l}\text { Sadeghi et al. (2014, 2016a, b, c, 2017a, b), Hazbavi and Sadeghi (2016), } \\
\text { Kheirfam et al. (2017a, b), Sharifi Moghaddam et al. (2014), Ghavimi Panah et al. (2017), } \\
\text { Kiani-Harchegani et al. (2017) }\end{array}$ \\
\hline 5 & 427 & 466 & M. H. Mohammadi & mhmohmad@ut.ac.ir & Unpublished data \\
\hline 6 & 467 & 505 & F. Meunier & felicien.meunier@gmail.com & Unpublished data \\
\hline 7 & 506 & 541 & N. Sephrnia & n.sepehrnia@gmail.com & Sepehrnia et al. $(2016,2017)$ \\
\hline 8 & 569 & 817 & D. Moret-Fernández & david@eead.csic.es & Unpublished data \\
\hline 9 & 818 & 940 & M. Vafakhah & vafakhah@modares.ac.ir & Kavousi et al. (2013), Fakher Nikche et al. (2014) \\
\hline 10 & 941 & 1060 & A. Cerdà & artemio.cerda@uv.es & Unpublished data \\
\hline 11 & 1061 & 1079 & J. Rodrigo-Comino & rodrigo-comino@uma.es & Rodrigo-Comino et al. $(2016,2018)$ \\
\hline 12 & 1080 & 1112 & H. Asadi & ho.asadi@ut.ac.ir & Nikghalpour et al. (2016) \\
\hline 13 & 1113 & 1119 & K. Bohne & klaus.bohne@uni-rostock.de & Unpublished data \\
\hline 14 & 1120 & 1125 & L. Mao & leoam@126.com & Mao et al. (2008b, 2016) \\
\hline 15 & 1126 & 1166 & L. Lichner & lichner@uh.savba.sk & Dušek et al. (2013), Lichner et al. (2011, 2012, 2013) \\
\hline 16 & 1167 & 1210 & M. V. Ottoni & marta.ottoni@cprm.gov.br & Oliveira (2005) \\
\hline $\begin{array}{l}17 \\
18\end{array}$ & $\begin{array}{l}1211 \\
4476\end{array}$ & $\begin{array}{l}1420 \\
4485\end{array}$ & R. Sándor & sandor.rencsi@gmail.com & Fodor et al. (2011), Sándor et al. (2015) \\
\hline 19 & 1508 & 1519 & A. Stanley & ajayistan@gmail.com & Igbadun et al. (2016), Othman and Ajayi (2016) \\
\hline 20 & 1520 & 1521 & A. R. Vaezi & vaezi.alireza@gmail.com & Unpublished data \\
\hline 21 & 1522 & 1536 & A. Albalasmeh & aalbalasmeh@just.edu.jo & Gharaibeh et al. (2016) \\
\hline 22 & 1537 & 1578 & D. Machiwal & dmachiwal@rediffmail.com & Machiwal et al. (2006, 2017), Ojha et al. (2013) \\
\hline 23 & 1579 & 1592 & H. Emami & hemami@um.ac.ir & Fakouri et al. $(2011 \mathrm{a}, \mathrm{b})$ \\
\hline 24 & 1593 & 1895 & J. Mertens & jan.mertens@engie.com & Mertens et al. $(2002,2004,2005)$ \\
\hline 25 & 1896 & 2115 & D. Jacques & diederik.jacques@sckcen.be & Jacques (2000), Jacques et al. (2002) \\
\hline 26 & 2116 & 2139 & J. Votrubova & jana.votrubova@fsv.cvut.cz & Votrubova et al. (2017) \\
\hline 27 & 2140 & 2143 & J. Batlle-Aguilar & jorbat1977@hotmail.com & Batlle-Aguilar et al. (2009) \\
\hline 28 & 2144 & 2179 & R. A. Armindo & rarmindo@ufpr.br & Unpublished data \\
\hline 29 & 2180 & 2209 & S. Werner & steffen.werner@rub.de & Unpublished data \\
\hline 30 & 2210 & 2255 & S. Zacharias & steffen.zacharias@ufz.de & Unpublished data \\
\hline 31 & 2256 & 2281 & S. Shutaro & sshiraki@affrc.go.jp & Unpublished data \\
\hline 32 & 2282 & 2304 & T. Saito & tadaomi@muses.tottori-u.ac.jp & Saito et al. (2016) \\
\hline 33 & 2305 & 2354 & R. Taghizadeh-M. & rh_taghizade@yahoo.com & Unpublished data \\
\hline 34 & 2355 & 2356 & & & \\
\hline 35 & 3644 & 3647 & W. G. Teixeira & wenceslau.teixeira@embrapa.br & Teixeira et al. (2014) \\
\hline 36 & 2357 & 2436 & Y. Zhao & yzhaosoils@gmail.com & Zhao et al. (2011) \\
\hline 37 & 2437 & 2475 & A. A. Moosavi & aamousavi@gmail.com & Unpublished data \\
\hline 38 & 2476 & 2552 & Y. A. Pachepsky & yakov.pachepsky@ars.usda.gov & Rawls et al. (1976) \\
\hline 39 & 2553 & 2643 & A. Panagopoulos & panagopoulosa@gmail.com & Hatzigiannakis and Panoras (2011) and unpublished data \\
\hline 40 & 2644 & 2649 & B. Clothier & brent.clothier@plantandfood.co.nz & Al Yamani et al. (2016) \\
\hline 41 & 2650 & 2710 & C. Coctollonat & (1) & \\
\hline 42 & 3507 & 3597 & C. Castellano & ccastellanonavarro@gmail.com & Unpublished data \\
\hline 43 & 2711 & 2756 & F. Becker & fabian.becker@fu-berlin.de & Unpublished data \\
\hline 44 & 2757 & 2765 & I. Vogeler & iris.vogeler@plantandfood.co.nz & Vogeler et al. (2006), Cichota et al. (2013) \\
\hline 45 & 2766 & 2788 & R. Morbidelli & renato.morbidelli@unipg.it & Morbidelli et al. (2017) \\
\hline 46 & 2789 & 2832 & S. Giertz & sgiertz@uni-bonn.de & Giertz et al. (2005) \\
\hline 47 & 2833 & 2868 & T. Vogel & vogel@fsv.cvut.cz & Vogel and Cislerova (1993) \\
\hline 48 & 2869 & 2948 & W. Cornelis & wim.cornelis@ugent.be & Pulido Moncada et al. (2014), Rezaei et al. (2016a, b) \\
\hline 49 & 2949 & 3386 & Y. Coquet & yves.coquet@univ-orleans.fr & Coquet (1996), Coquet et al. (2005), Chalhoub et al. (2009) \\
\hline 50 & 3705 & 3709 & Y. Coquet & yves.coquet@unı-orleans.fr & Coquet (1996), Coquet et al. (2005), Chalhoub et al. (2009) \\
\hline 51 & 3387 & 3506 & B. Mohanty & bmohanty@tamu.edu & Dasgupta et al. (2006) \\
\hline 52 & 3598 & 3643 & D. J. Reinert & dalvan@ufsm.br & Mallmann (2017) \\
\hline 53 & 3648 & 3657 & M. R. Pahlavan Rad & pahlavanrad@gmail.com & Pahlavan-Rad (2017) \\
\hline 54 & 3658 & 3680 & T. Saito & tadaomi@muses.tottori-u.ac.jp & Unpublished data \\
\hline 55 & 3681 & 3704 & & & \\
\hline 56 & 4497 & 4505 & X. Li & xyli@bnu.edu.cn & Li et al. (2013), Hu et al. (2016) \\
\hline 57 & 3710 & 3745 & Y. Bamutaze & yazidhibamutaze@gmail.com & Unpublished data \\
\hline 58 & 3746 & 3833 & & & \\
\hline 59 & 3907 & 4011 & I. Braud & isabelle.braud@irstea.fr & Gonzalez-Sosa et al. (2010), Braud (2015), Braud and Vandervaere (2015) \\
\hline 60 & 3834 & 3874 & M. R. Mosaddeghi & mosaddeghi@yahoo.com & Unpublished data \\
\hline 61 & 3875 & 3906 & S. B. Mousavi & b_mosavi2000@yahoo.com & Unpublished data \\
\hline 62 & 4012 & 4026 & M. Pulido & manpufer@hotmail.com & Unpublished data \\
\hline 63 & 4027 & 4457 & & & Unpublished data \\
\hline & 4458 & 4475 & F. P. Roberts & frapar@ceh.ac.uk & Robinson et al. $(2016,2017)$ \\
\hline 64 & 4486 & 4496 & T. Picciafuoco & picciafuoco@hydro.tuwien.ac.at & Morbidelli et al. (2017) \\
\hline 65 & 4880 & 4886 & M. A. Liebig & mark.liebig@ars.usda.gov & Liebig et al. (2004) \\
\hline 66 & 4887 & 4936 & Y. Zeng & y.zeng@utwente.nl & Zhao et al. $(2017,2018)$ \\
\hline 67 & 4937 & 5018 & L. Lassabatere & laurent.lassabatere@entpe.fr & Lassabatere et al. (2010), Yilmaz et al. (2010), Coutinho et al. (2016) \\
\hline 68 & 5019 & 5023 & I. Eskandari & eskandari1343@yahoo.com & Unpublished data \\
\hline
\end{tabular}


Table 3. Description of the variables listed in the database.

\begin{tabular}{|c|c|c|}
\hline Column & Supplies & Dimension \\
\hline Code & \multicolumn{2}{|l|}{ Dataset identifier with 4 digits from 0001 to 5023} \\
\hline Clay & Mass of soil particles, $<0.002 \mathrm{~mm}$ & $\%$ \\
\hline Silt & Mass of soil particles, $>0.002$ and $<0.05 \mathrm{~mm}$ & $\%$ \\
\hline Sand & Mass of soil particle, $>0.05$ and $<2 \mathrm{~mm}$ & $\%$ \\
\hline Texture & \multicolumn{2}{|c|}{$\begin{array}{l}\text { 1: sand; } 2 \text { : loamy sand; } 3 \text { : sandy loam; } 4 \text { : sandy clay loam; } 5 \text { : sandy clay; } 6 \text { : loam; } \\
\text { 7: silt loam; } 8 \text { : silt; } 9 \text { : clay loam; } 10: \text { silty clay loam; } 11: \text { silty clay; } 12 \text { : clay. }\end{array}$} \\
\hline Gravel & Mass of particles larger than $2 \mathrm{~mm}$ & $\%$ \\
\hline$d_{\mathrm{g}}$ & Geometric mean diameter & $\mathrm{mm}$ \\
\hline$S_{\mathrm{g}}^{0}$ & \multicolumn{2}{|l|}{ Standard deviation of soil particle diameter } \\
\hline $\mathrm{OC}$ & Soil organic carbon content & $\%$ \\
\hline$D_{\mathrm{b}}$ & Soil bulk density & $\mathrm{g} \mathrm{cm}^{-3}$ \\
\hline$D_{\mathrm{p}}$ & Soil particle density & $\mathrm{g} \mathrm{cm}^{-3}$ \\
\hline$K_{\text {sat }}$ & Soil saturated hydraulic conductivity & $\mathrm{cm} \mathrm{h}^{-1}$ \\
\hline$\theta_{\text {sat }}$ & Saturated volumetric soil water content & $\mathrm{cm}^{3} \mathrm{~cm}^{-3}$ \\
\hline$\theta_{\mathrm{i}}$ & Initial volumetric soil water content & $\mathrm{cm}^{3} \mathrm{~cm}^{-3}$ \\
\hline FC & Soil water content at field capacity & $\mathrm{cm}^{3} \mathrm{~cm}^{-3}$ \\
\hline PWP & Soil water content at permanent wilting point $(1500 \mathrm{kPa})$ & $\mathrm{cm}^{3} \mathrm{~cm}^{-3}$ \\
\hline$\theta_{\mathrm{r}}$ & Residual volumetric soil water content & $\mathrm{cm}^{3} \mathrm{~cm}^{-3}$ \\
\hline WAS & Wet-aggregate stability & $\%$ \\
\hline MWD & Aggregates mean weight diameter & $\mathrm{mm}$ \\
\hline GMD & Aggregates geometric mean diameter & $\mathrm{mm}$ \\
\hline $\mathrm{EC}$ & Soil electrical conductivity & $\mathrm{dS} \mathrm{m} \mathrm{m}^{-1}$ \\
\hline $\mathrm{pH}$ & Soil acidity & - \\
\hline Gypsum & Soil gypsum content & $\%$ \\
\hline CCE & Soil calcium carbonate equivalent & $\%$ \\
\hline CEC & Soil cation exchange capacity & $\mathrm{Cmol}_{\mathrm{c}} \mathrm{kg}^{-1}$ \\
\hline SAR & Soil sodium adsorption ratio & - \\
\hline DiscRadius & Applied disc radius (if any) & $\mathrm{mm}$ \\
\hline \multirow[t]{2}{*}{ Instrument } & \multicolumn{2}{|l|}{ Applied instruments for infiltration measurement: } \\
\hline & \multicolumn{2}{|c|}{$\begin{array}{l}\text { 1: double ring; } 2 \text { : single ring; } 3 \text { : rainfall simulator; 4: Guelph permeameter; 5: disc infiltrometer; } \\
\text { 6: micro-infiltrometer; 7: mini-infiltrometer; 8: Aardvark permeameter; 9: linear source method; } \\
\text { 10: point source method; 11: hood infiltrometer; 12: tension infiltrometer; 13: BEST method. }\end{array}$} \\
\hline Vegetation cover & & $\%$ \\
\hline Land use & \multicolumn{2}{|l|}{ Dominant land-use or land cover type of the experimental site } \\
\hline Rainfall intensity & Simulated rain intensity & $\mathrm{mm} \mathrm{h}^{-1}$ \\
\hline Slope & The mean slope of the soil surface & $\%$ \\
\hline Treatment & \multicolumn{2}{|l|}{ Applied treatment in experimental site } \\
\hline Crust & \multicolumn{2}{|l|}{ Yes: existence of crust. No: no crust layer. } \\
\hline Sand contact layer & \multicolumn{2}{|c|}{ Yes: sand contact layer is applied during infiltration measurement. No: no sand contact layer. } \\
\hline
\end{tabular}

tem (Fig. 4), in total, 35 WRB reference soil subgroups are included among experimental sites, where $55 \%$ of the experimental sites comprised four subgroup classes of Haplic Acrisols (8\%), Haplic Luvisols (11\%), Haplic Calcisols (15\%), and Haplic Cambisols (21\%). A total of 29 soil suborders classes of USDA soil taxonomy are included in this study (Fig. 5) with Udalfs (9\%), Orthents (9\%), and Ustolls (9\%). Thus, the wide spatial and temporal distribution of infiltration data from this database provides a comprehensive view of the infiltration characteristics of many soils in the world which can be used in future studies.

\subsection{Analysis of the database using soil properties}

Textural information (clay, silt, and sand content) is available for 3842 out of 5023 collected infiltration curves $(\sim 76 \%)$. The infiltration measurements cover nearly all soil textural classes according to the USDA classification, except for the sandy clay and silt textural class (Fig. 6), which makes the SWIG database a valuable data source for comprehensive studies. To complete the large dataset, the open-access SWIG database might be amended with information regarding those soils poorly or altogether unrepresented by the existing database, including those not usually considered by infiltration studies, such as soils with extremely high stone 


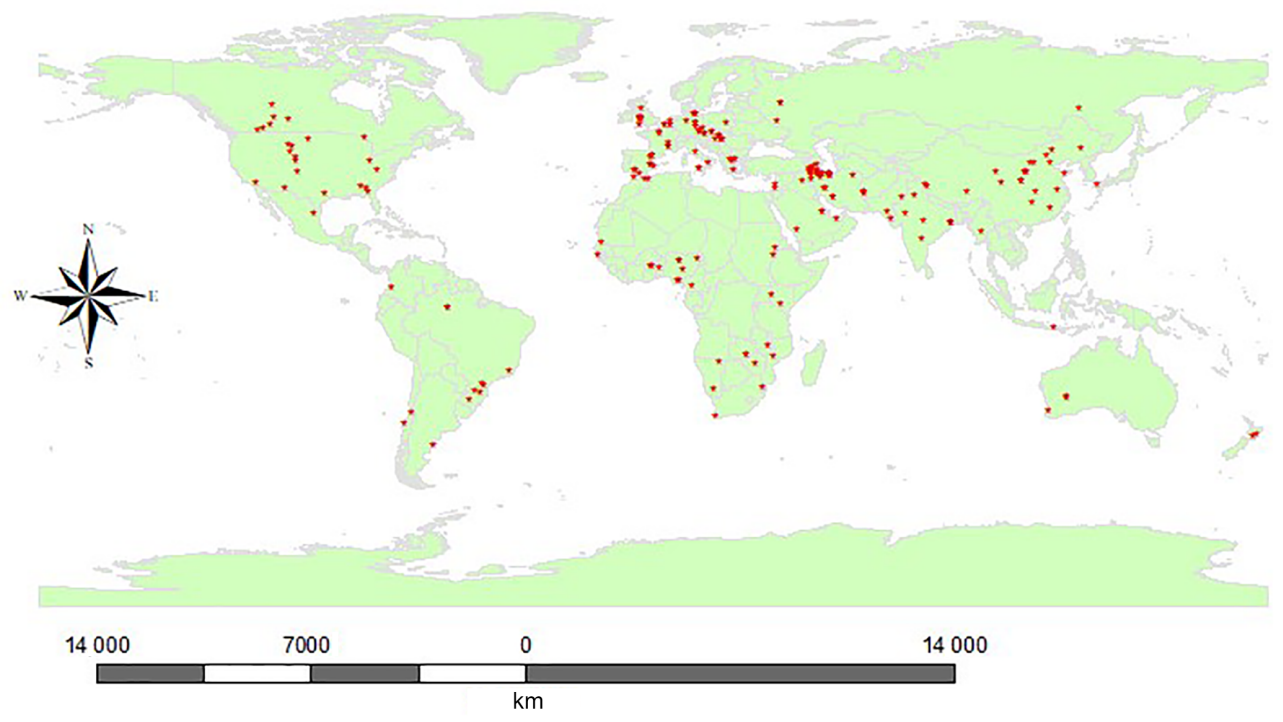

Figure 2. Global distribution of infiltration measuring sites that were included in the database.

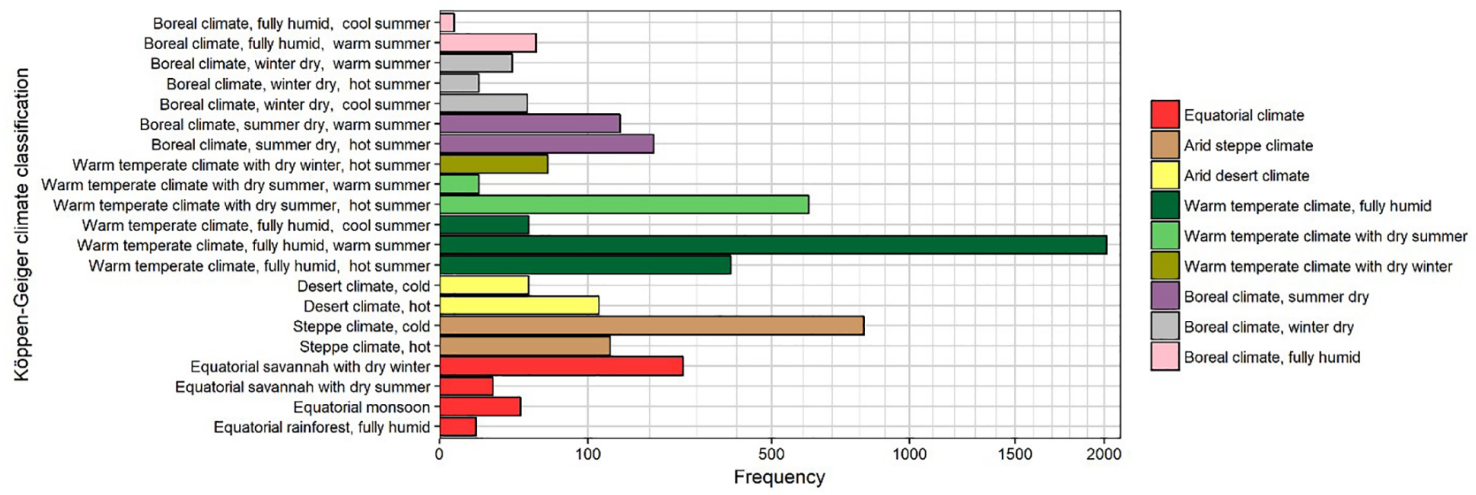

Figure 3. Number of samples by Köppen-Geiger climatic zones (Rubel et al., 2017; Kottek et al., 2006).

content (Poesen, 2018). Loam, sandy loam, silty loam, and clay loam contributed with $19,18,14$, and $13 \%$ (Table 5) to the infiltration measurements, respectively. Table 5 shows that infiltration measurements are almost equally distributed among textures when these are categorized in three major classes: course- (1092), medium- (1238), and fine- to moderately fine-textured soils (1447). Table 6 reports on the soil properties that are available in the SWIG database and it gives some simple statistics such as mean, minimum, maximum, median, and coefficient of variation. Bulk density (available for $66 \%$ of infiltration measurements) and organic carbon content (available for $62 \%$ of infiltration measurements) are two other soil properties besides texture that have the highest frequency of availability. Saturated hydraulic conductivity, initial soil water content, saturated soil water content, calcium carbonate equivalent, electrical conductivity, and $\mathrm{pH}$ are available in 22 to $38 \%$ of infiltration data. The other soil properties have a frequency lower than $10 \%$.

\subsection{Infiltration measurements in the SWIG database}

Different instruments were used to measure soil water infiltration (Table 8). About $32 \%$ (1595 out of 5023) of the measurements were carried out using different types of ring infiltrometers. The most frequently used methods are the disc infiltrometer methods (disc, mini-disc, and micro-disc, hood, and tension infiltrometers), which have been used in about $51 \%$ of the experiments. About $5 \%$ of the data were submitted to the database without specifying the measurement method (251 infiltration tests) and around $12 \%$ of the measurements were carried out with other methods not listed above (Table 7).

\subsection{Land use classes represented in the SWIG database}

Land use is known to potentially impact soil structure and then water infiltration into soils (e.g., Ilstedt et al., 2007; Wa- 
Table 4. Countries and the number of data sources $(n)$ contributing to the database.

\begin{tabular}{lrll}
\hline Country & $n$ & Country & $n$ \\
\hline Iran & 38 & Slovakia & 2 \\
China & 23 & South Africa & 2 \\
USA & 15 & Sudan & 2 \\
Brazil & 9 & Zambia & 2 \\
Spain & 9 & Argentina & 1 \\
France & 9 & Australia & 1 \\
Germany & 8 & Benin & 1 \\
India & 8 & Cameroon & 1 \\
Canada & 7 & Colombia & 1 \\
United Kingdom & 7 & Indonesia & 1 \\
Hungary & 6 & Iraq & 1 \\
Nigeria & 6 & Japan & 1 \\
Greece & 5 & Jordan & 1 \\
Belgium & 4 & Kenya & 1 \\
Italy & 4 & Lebanon & 1 \\
Czech Republic & 3 & Malawi & 1 \\
Saudi Arabia & 3 & Mexico & 1 \\
Australia & 2 & Mozambique & 1 \\
Austria & 2 & Myanmar & 1 \\
Chile & 2 & Netherland & 1 \\
Ghana & 2 & Poland & 1 \\
Morocco & 2 & Scotland & 1 \\
Namibia & 2 & Tanzania & 1 \\
New Zealand & 2 & Telangana & 1 \\
Pakistan & 2 & UAE & 1 \\
Russia & 2 & Uganda & 1 \\
Senegal & 2 & Zimbabwe & 1 \\
\hline & & &
\end{tabular}

Table 5. Number of soils in each soil USDA textural class for which infiltration data are included in the database.

\begin{tabular}{llr}
\hline Group & Soil texture class & Availability \\
\hline Coarse-textured soils & 1092 & \\
\hline & Sand & 291 \\
& Loamy sand & 111 \\
& Sandy loam & 690 \\
\hline Medium-textured soils & 1238 & \\
& Loam & 716 \\
& Silt loam & 522 \\
& Silt & 0 \\
\hline Fine- to moderately & & 1476 \\
fine-textured soil & & \\
\hline & Clay loam & 514 \\
& Clay & 352 \\
& Silty clay loam & 253 \\
& Sandy clay loam & 226 \\
& Silty clay & 131 \\
& Sandy clay & 0 \\
\hline
\end{tabular}

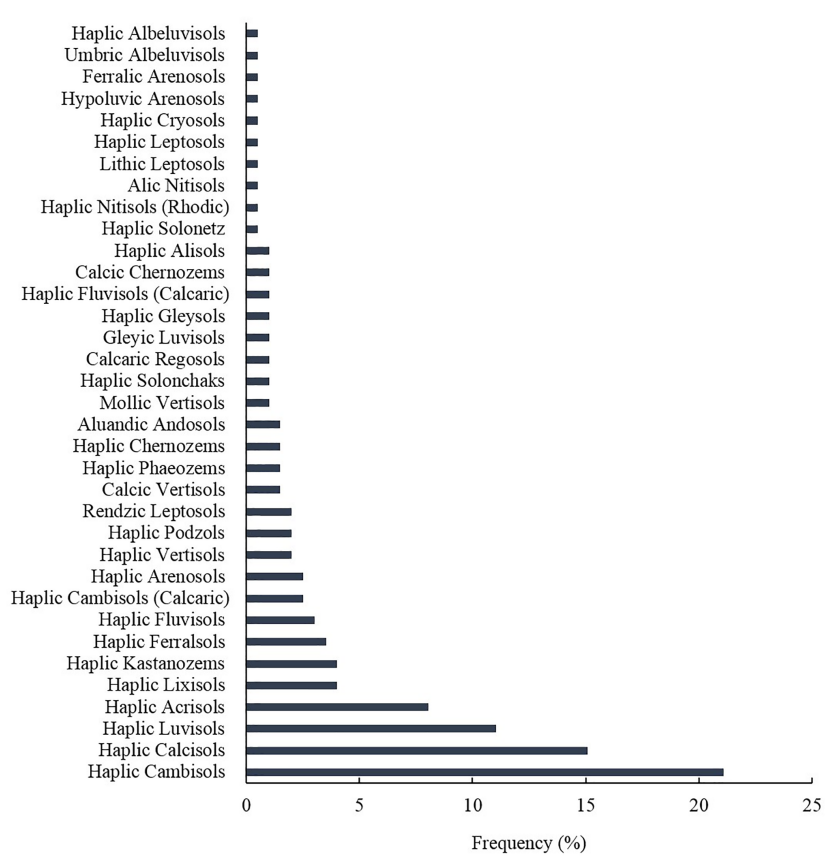

Figure 4. Frequency of WRB reference soil subgroups in experimental sites derived from SoilGrids (Hengl et al., 2017).

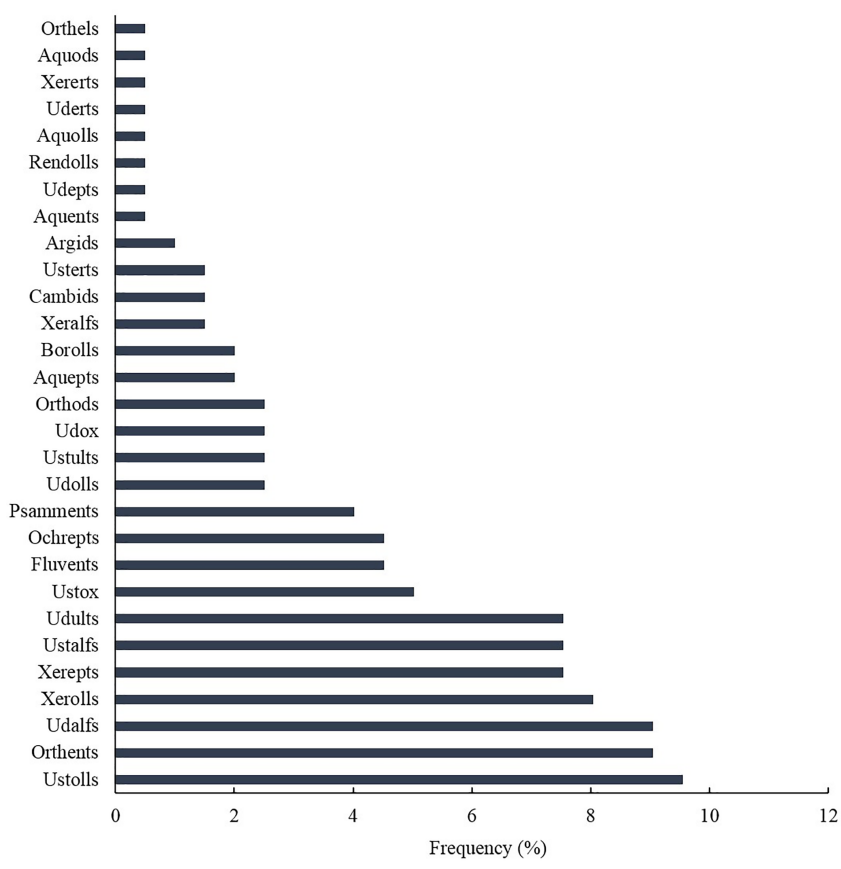

Figure 5. Frequency of USDA soil suborders in experimental sites derived from SoilGrids (Hengl et al., 2017). 

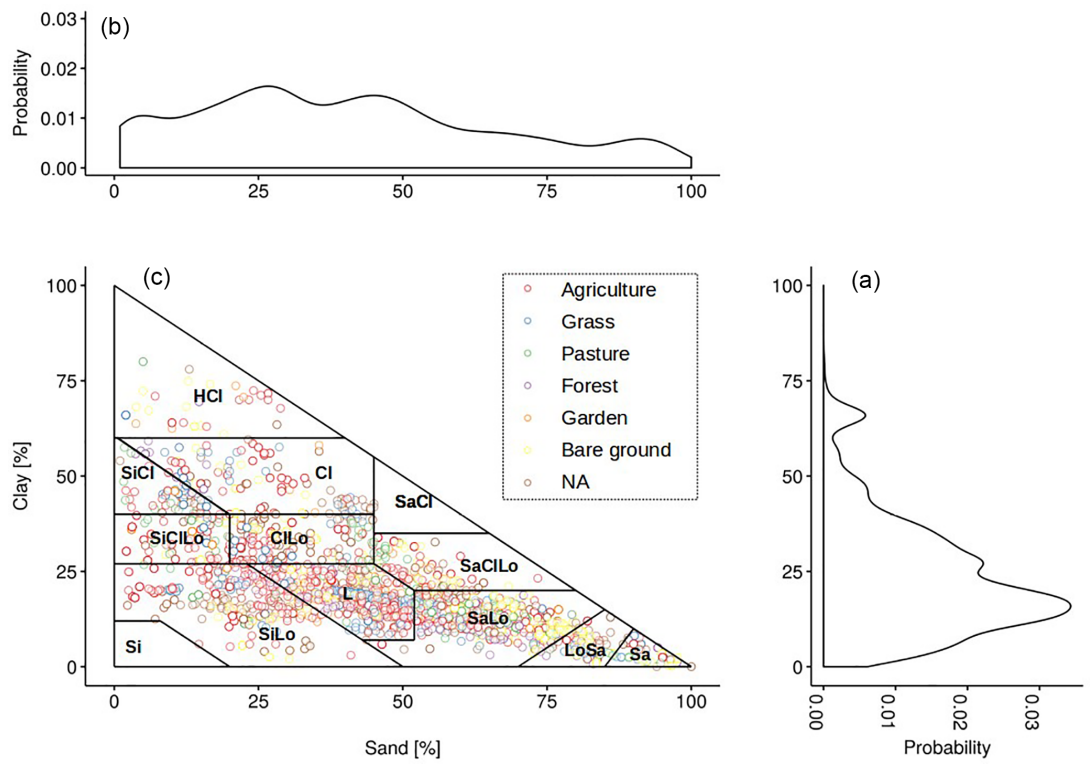

Figure 6. Textural distribution of soils (c) and probability density functions of clay (a) and sand (b) particles (plotted on the USDA textural triangle) for which infiltration data are included in the database. Dots are colored according to their corresponding land use. $\mathrm{HCl}$ : highly clayey; SiCl: silty clay; Cl: clay; SiClLo: silty clay loam; ClLo: clay loam; SaCl: sandy clay; SaClLo: sandy clay loam; L: loam; Si: silty; SiLo: silty loam; SaLo: sandy loam; LoSa: loamy sand; and Sa: sandy.

Table 6. Soil properties, number of data entries in the database (out of 5023 soil water infiltration curves in total), and their statistical description.

\begin{tabular}{|c|c|c|c|c|c|c|c|}
\hline Soil properties & Availability & $\operatorname{Fr}(\%)$ & Mean & Min & $\operatorname{Max}$ & Median & $\mathrm{CV}(\%)$ \\
\hline Clay $(\%)$ & 3842 & 76 & 24 & 0 & 80 & 20 & 64 \\
\hline Silt $(\%)$ & 3842 & 76 & 36 & 0 & 82 & 37 & 52 \\
\hline Sand $(\%)$ & 3842 & 76 & 41 & 1 & 100 & 38 & 63 \\
\hline Bulk density $\left(\mathrm{g} \mathrm{cm}^{-3}\right)$ & 3295 & 66 & 1.32 & 0.14 & 2.81 & 1.35 & 20 \\
\hline Organic carbon $(\%)$ & 3102 & 62 & 3 & 0 & 88 & 1 & 200 \\
\hline Saturated hydraulic cond. $\left(\mathrm{cm} \mathrm{h}^{-1}\right)$ & 1895 & 38 & 41 & 0 & 3004 & 3 & 426 \\
\hline Initial soil water content $\left(\mathrm{cm}^{3} \mathrm{~cm}^{-3}\right)$ & 1569 & 31 & 0.17 & 0 & 0.63 & 0.14 & 68 \\
\hline Saturated soil water content $\left(\mathrm{cm}^{3} \mathrm{~cm}^{-3}\right)$ & 1400 & 28 & 0.44 & 0.01 & 0.87 & 0.45 & 24 \\
\hline Carbonate calcium equivalent $(\%)$ & 1399 & 28 & 14 & 0 & 56 & 8 & 101 \\
\hline Electrical conductivity $\left(\mathrm{dS} \mathrm{m}^{-1}\right)$ & 1113 & 22 & 25 & 0 & 358 & 1 & 249 \\
\hline 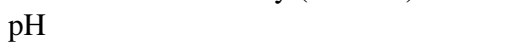 & 1081 & 22 & 7.4 & 4.7 & 8.6 & 7.6 & 12 \\
\hline Particle density $\left(\mathrm{g} \mathrm{cm}^{-3}\right)$ & 438 & 9 & 2.52 & 1.73 & 2.97 & 2.56 & 9 \\
\hline Gypsum (\%) & 380 & 8 & 4 & 0 & 49 & 3 & 137 \\
\hline Cation exchange capacity $\left(\mathrm{cmol}_{\mathrm{c}} \mathrm{kg}^{-1}\right)$ & 357 & 7 & 17 & 3 & 26 & 18 & 21 \\
\hline Wet-aggregate stability (\%) & 309 & 6 & 61 & 5 & 96 & 63 & 37 \\
\hline Residual soil water content $\left(\mathrm{cm}^{3} \mathrm{~cm}^{-3}\right)$ & 263 & 5 & 0.10 & 0.001 & 0.38 & 0.06 & 86 \\
\hline Mean weight diameter (mm) & 258 & 5 & 1 & 0.10 & 2.75 & 1.0 & 54 \\
\hline Gravel (\%) & 243 & 5 & 18 & 0 & 92 & 15 & 84 \\
\hline Sodium adsorption ratio & 156 & 3 & 5 & 0 & 89 & 1 & 351 \\
\hline Soil water content at $\mathrm{FC}\left(\mathrm{cm}^{3} \mathrm{~cm}^{-3}\right)$ & 74 & 1 & 0.28 & 0.12 & 0.54 & 0.27 & 34 \\
\hline Soil water content at PWP $\left(\mathrm{cm}^{3} \mathrm{~cm}^{-3}\right)$ & 64 & 1 & 0.18 & 0.05 & 0.36 & 0.20 & 47 \\
\hline Geometric mean diameter (mm) & 73 & 1 & 0.6 & 0.4 & 0.8 & 0.6 & 18 \\
\hline
\end{tabular}

Fr: frequency (\%), Min: minimum, Max: maximum, CV: coefficient of variation. 
Table 7. Instruments used to measure soil infiltration curves.

\begin{tabular}{llr}
\hline Instrument/method used & & Infiltration curves \\
Ring & Double ring & 828 \\
& Single ring & 570 \\
& Beerkan (BEST) & 197 \\
\hline Overall & Disc & 1595 \\
\hline \multirow{2}{*}{ Infiltrometer } & Mini-disc & 607 \\
& Micro-disc & 1140 \\
& Hood & 36 \\
Overall & Tension & 23 \\
\hline \multirow{2}{*}{ Permeameter } & & 752 \\
\hline Overall & Guelph & 2558 \\
\hline Rainfall simulator & Aardvark & 181 \\
Linear source method & & 50 \\
Point source method & & 231 \\
Not reported & & 374 \\
\hline Sum & & 10 \\
\hline
\end{tabular}

Table 8. Number of infiltration curves with a given land use type.

\begin{tabular}{lrlr}
\hline Land use & $n$ & Land use & $n$ \\
\hline Agriculture & 2019 & Vineyards & 22 \\
Grassland & 821 & Upland & 11 \\
Pasture & 229 & Pure sand & 10 \\
Forest & 204 & Brushwood & 6 \\
Garden & 152 & Road & 5 \\
Bare & 99 & Agro-pastoral & 4 \\
Urban soils & 82 & Park & 3 \\
Savannah & 41 & Salt-marsh soil & 3 \\
Abandoned farms & 39 & Afforestation & 2 \\
Idle & 32 & Campus & 2 \\
Shrub & 30 & Residential & 2 \\
\hline Available & 3818 & Unknown & 1205 \\
\hline
\end{tabular}

terloo et al., 2007). Consequently, we collected information on the type of land use at all experimental sites where available. In general, the type of land use was reported in 3818 out of 5023 infiltration curves $(\sim 76 \%)$ and this information is reported in the Metadata dataset. For simplicity, we grouped all reported land use types into 22 major groups (Table 8). A frequency analysis showed that agricultural land use, i.e., cropped land, irrigated land, dryland, and fallow land, is the most frequently reported land use in the database with about $53 \%$ (2019 out of 3818) of all land uses. With $22 \%$, grasslands are the second most frequently represented land use type. Pasture with $6 \%$ and forest with $5 \%$ are ranked as the third- and fourth-largest reported land use types. The 18 re- maining land use types all together cover only 545 experimental sites (less than $15 \%$ ).

\subsection{Estimating infiltration parameters from infiltration measurements}

In order to predict infiltration parameters from infiltration measurements, we classified the SWIG database infiltration curves in two groups: (i) infiltration curves that were obtained under the assumption of 1-D infiltration and (ii) infiltration curves that were obtained under 3-D flow conditions. We fitted the three-parameter infiltration equation of Philip (Kutílek and Krejča, 1987), Eq. (6), to the 1-D experimental data and the simplified form of Haverkamp et al. (1994), Eq. (7), to the 3-D experimental data:

$$
\begin{aligned}
& I_{1-\mathrm{D}}=S t^{\frac{1}{2}}+A_{1} t+A_{2} t^{\frac{3}{2}}, \\
& I_{3-\mathrm{D}}=S \sqrt{t}+\left[\frac{2-\beta}{3} K_{\text {sat }}+\frac{\gamma S^{2}}{R_{\mathrm{D}}\left(\theta_{\mathrm{s}}-\theta_{\mathrm{i}}\right)}\right] t .
\end{aligned}
$$

We reduced the number of parameters in Eq. (6) by defining $A_{1}=0.33 \times K_{\text {sat }}$ (Philip, 1957) and $A_{2}=A$ where $A$ was assumed to be a constant. In Eq. (7), we put $\beta=0.6$ (AnguloJaramillo et al., 2000) and the second term between brackets on the right-hand side was assumed to be a constant. Therefore, we simplified the equations as follows:

$$
\begin{aligned}
& I_{1-\mathrm{D}}=S t^{\frac{1}{2}}+0.33 K_{\mathrm{sat}} t+A t^{\frac{3}{2}}, \\
& I_{3-\mathrm{D}}=S \sqrt{t}+0.47 K_{\mathrm{sat}} t+A t .
\end{aligned}
$$

In our analysis, we assumed that double-ring infiltrometer measurements result in 1-D infiltration conditions, while the different types of disc infiltration and single-ring infiltrometer measurements lead to 3-D flow conditions that can be captured by Eq. (9). As 1-D or 3-D infiltration conditions are not guaranteed for measurements made with rainfall simulators, Guelph permeameters, Aardvark permeameters, linear and point source methods, and hood infiltrometer measurements, these infiltration curves were not considered in our first analysis. By excluding these methods, 596 infiltration curves were excluded from the fitting to Eqs. (8) and (9). In addition, 251 infiltration curves were also excluded from the fitting to Eqs. (8) and (9) as no indication was available on the measurement method used. In total, 4178 infiltration curves were included in our analysis, of which 828 infiltration curves reflected 1-D and 3350 were considered as the results of 3-D infiltration. As no sufficient information was available on the properties of the sand contact layer, we did not correct 3-D infiltration measurements. Finally, the selected infiltration curves were fitted to Eq. (8) or (9) using the lsqnonlin command in Matlab ${ }^{\mathrm{TM}}$.

The fitting results of Eq. (8) to the single infiltrometer data are shown in Table 9. $R^{2}$ values were higher than 0.9 in $97 \%$ of the cases and higher than 0.99 in $77 \%$ of the cases. Fitting Eq. (9) to the 3-D infiltration curves data, $R^{2}$ values higher 
than 0.9 and 0.99 were obtained in 94 and $68 \%$ of the cases, respectively. The statistics for the fitting process as well as the fitted parameters of two mentioned models are reported in the SWIG database in an additional dataset labeled "Statistics". For infiltration curves excluded from the analysis, an empty cell is reported.

The average values of estimated $K_{\text {sat }}$ and sorptivity $(S)$, using Eq. (8) or (9) as well as measured $K_{\text {sat }}$ for different soil texture classes extracted from the current database, are reported in Table 10. The measured values of $K_{\text {sat }}$ were obtained by other means by the contributors and tabulated in the SWIG database. More detailed information of how $K_{\text {sat }}$ was calculated in individual cases can be found in the references linked to those data points. Comparison between estimated ( $\left.K_{\text {sat-es }}\right)$ and measured $\left(K_{\text {sat-m }}\right)$ values of $K_{\text {sat }}$ (Table 10$)$ reveals that there is reasonably good agreement between measurements and estimation, except for loamy sand (with mean $K_{\text {sat-es }}=62 \mathrm{~cm} \mathrm{~h}^{-1}$ vs. $K_{\text {sat-m }}=25 \mathrm{~cm} \mathrm{~h}^{-1}$ ), sandy loam (with mean $K_{\text {sat-es }}=32 \mathrm{~cm} \mathrm{~h}^{-1}$ vs. $K_{\text {sat-m }}=41 \mathrm{~cm} \mathrm{~h}^{-1}$ ), silt loam (with mean $K_{\text {sat-es }}=27 \mathrm{~cm} \mathrm{~h}^{-1}$ vs. $K_{\text {sat-m }}=3 \mathrm{~cm} \mathrm{~h}^{-1}$ ), and silty clay (with mean $K_{\text {sat-es }}=26 \mathrm{~cm} \mathrm{~h}^{-1}$ vs. $K_{\text {sat-m }}=$ $45 \mathrm{~cm} \mathrm{~h}^{-1}$ ) textural classes. However, the only significant difference between measured and estimated $K_{\text {sat }}$ values was found for the silt loam textural class (Table 10) applying an independent $t$ test.

We also compared our estimated $K_{\text {sat }}$ values from the infiltration measurements from the SWIG database with $K_{\text {sat }}$ values from databases that have been published in the literature (Table 11). The validity of our estimated $K_{\text {sat }}$ values is confirmed by comparing the order of magnitude of the difference between these values, and those tabulated in previous studies, for the various different soil classes. Some of these databases like that of Clapp and Hornberger (1978) and Cosby et al. (1984) have been used to parameterize land surface models. Most of the $K_{\text {sat }}$ values in the listed databases have been obtained from laboratory-scale measurements often performed on disturbed soil samples. In most of the reported databases $K_{\text {sat }}$ is controlled by texture, with the highest mean values obtained for the coarse-textured soils and the lowest mean values for the fine-textured soils. This is not the case for the $K_{\text {sat }}$ values obtained from the SWIG database. Clayey soils have a mean value that is similar to the coarser textured soils. This may be partly explained by the fact that the measurements collected in the SWIG database are obtained from field measurements on undisturbed soils. It was observed that the standard deviation of $K_{\text {sat }}$ in the SWIG database is typically larger than the standard deviations obtained from the databases in the literature. This indicates that texture is apparently not the most important control on $K_{\text {sat }}$ values. However, one would also pose that much of the lack of correlation between soil texture and predicted $K_{\text {sat }}$ from the SWIG database is related to the lack of soil structural information, such as macro porosity quantification or other possible soil attributes. Indeed, many of the datasets presented in our paper on saturated and near-saturated flow can be used to infer the state of the soil's structure, namely its macroporosity, by using the slope of the near-saturated conductivity curve, via Philip's "flow-weighted mean poresize" analysis. White and Sully (1987) have discussed this in a great detail. Zhang et al. (2015) is another example of where tension infiltrometers can be used to describe the temporal dynamics of the macroporosity which characterizes soil structure. This could inspire researchers to collect such information when conducting additional soil infiltration measurements and include this in the database in the future. This finding indicates that present parameterization in current land surface models, which are mainly based on texture, may severely underestimate the variability of $K_{\text {sat }}$. In addition, it shows that also mean values are not dominantly controlled by textural properties. Other land surface properties such as land use and crusting may, in fact, be much more important.

\subsection{Exploring the SWIG database using principal component analysis}

In order to demonstrate the potential of the SWIG database for analyzing infiltration data and for developing pedotransfer functions, principal component analysis (PCA) was performed and biplots were generated to show both the observations and the original variables in the principal component space (Gabriel, 1971).

In a biplot, positively correlated variables are closely aligned with each other and the larger the arrows the stronger the correlation. Arrows that are aligned in opposite directions are negatively correlated with each other and the magnitude of the arrows is again a measure of the strength of the correlation. Arrows that are aligned $90^{\circ}$ to each other show typically no correlation. Figures 7 and 8 show the results of two PCAs. The first PCA (Fig. 7) shows the relationship between soil textural properties, $S$ and $K_{\text {sat }}$, based on 3267 infiltration measurements. The first two principal components explain $74.5 \%$ of the variability in the data. Figure 7 shows a positive correlation between $K_{\text {sat }}$ and $S(0.527)$ and the largest values for both variables are found in clay soils. Clay content appears to only be weakly correlated with $K_{\text {sat }}$ and $S$ as is also shown by the correlation coefficients of 0.112 and 0.025 , respectively. Figure 8 shows the biplot of soil textural properties with $K_{\text {sat }}, S$, organic carbon content, and bulk density in the principal component space - based on 1910 infiltration measurements. The first two principal components still explain $55 \%$ of the variability. Neither $S$ nor $K_{\text {sat }}$ showed appreciable correlations with available soil properties. Only $K_{\text {sat }}$ and $S$ are correlated (arrows are aligned but small) with a value of 0.29 . Organic carbon and bulk density show a negative correlation with a calculated value equal to -0.51 . It also shows that, for example, the sandy clay loam textural class (yellow dots) shows a wide spread in organic matter content and bulk densities. These analyses show that the examined basic soil properties do not contain enough informa- 
Table 9. Accuracy analysis of empirical models fitted to experimental data of infiltration.

\begin{tabular}{|c|c|c|c|c|c|c|c|c|c|c|c|}
\hline \multirow{2}{*}{ Infiltration type } & \multirow[t]{2}{*}{$n$} & \multicolumn{4}{|c|}{$R^{2}$} & \multicolumn{4}{|c|}{ RMSE (cm) } & \multirow{2}{*}{$R^{2}>0.90$} & \multirow{2}{*}{$R^{2}>0.99$} \\
\hline & & Mean & Min & Max & SD & Mean & Min & Max & SD & & \\
\hline $1-\mathrm{D}$ & 828 & 0.985 & 0.529 & 1 & 0.049 & 0.900 & $1.3 \times 10^{-4}$ & 69.30 & 3.31 & 801 & 640 \\
\hline $3-\mathrm{D}$ & 3350 & 0.975 & 0.032 & 1 & 0.066 & 0.449 & $5.5 \times 10^{-12}$ & 98.95 & 2.95 & 3136 & 2276 \\
\hline All & 4178 & 0.977 & 0.032 & 1 & 0.063 & 0.538 & $5.5 \times 10^{-12}$ & 98.95 & 3.03 & 3937 & 2916 \\
\hline
\end{tabular}

SD: standard deviation.

Table 10. Estimated or measured average values of infiltration parameters for different textural classes extracted from the current database.

\begin{tabular}{|c|c|c|c|c|c|c|c|c|c|c|c|c|c|}
\hline \multirow[t]{3}{*}{ Texture class } & \multicolumn{7}{|c|}{ Estimated by Eq. (8) or (9) } & \multicolumn{4}{|c|}{ Measured } & \multicolumn{2}{|c|}{$\begin{array}{l}\text { Independent } t \text { test } \\
\text { between measured } \\
\text { and estimated } K_{\text {sat }}\end{array}$} \\
\hline & \multirow{2}{*}{$n^{\mathrm{a}}$} & \multicolumn{3}{|c|}{$S\left(\mathrm{~cm} \mathrm{~h}^{-0.5}\right)$} & \multicolumn{3}{|c|}{$K_{\text {sat }}\left(\mathrm{cm} \mathrm{h}^{-1}\right)$} & \multirow{2}{*}{$n^{\mathrm{a}}$} & \multicolumn{3}{|c|}{$K_{\text {sat }}\left(\mathrm{cm} \mathrm{h}^{-1}\right)$} & \multirow[t]{2}{*}{$\mathrm{df}$} & \multirow[t]{2}{*}{$t$ value } \\
\hline & & Mean & Median & SD & Mean & Median & SD & & Mean & Median & SD & & \\
\hline Sand & 291 & 2.3 & 0.26 & 4.3 & 42.2 & 15 & 134.5 & 229 & 43.6 & 24 & 149 & 518 & $0.10^{\mathrm{b}}$ \\
\hline Loamy sand & 92 & 10.6 & 5.7 & 17.5 & 61.4 & 10 & 173.2 & 63 & 24.6 & 8.2 & 72 & 153 & $1.59^{\mathrm{b}}$ \\
\hline Sandy loam & 500 & 9.2 & 2.95 & 15.7 & 32 & 3.1 & 94.5 & 424 & 41.2 & 5.7 & 166 & 922 & $1.05^{\mathrm{b}}$ \\
\hline Silt loam & 409 & 9.4 & 1.5 & 19.1 & 26.5 & 1.7 & 61.7 & 165 & 2.9 & 0.96 & 5.1 & 572 & $4.90^{\mathrm{c}}$ \\
\hline Loam & 583 & 7.9 & 2.4 & 12.9 & 7.8 & 0.28 & 26.7 & 270 & 4.9 & 1.18 & 13.7 & 851 & $1.69^{\mathrm{b}}$ \\
\hline Sandy clay loam & 185 & 5.9 & 2.1 & 8.6 & 7.4 & 1.4 & 12.8 & 84 & 5.4 & 2.24 & 6.9 & 267 & $1.35^{\mathrm{b}}$ \\
\hline Silty clay loam & 250 & 3.2 & 0.64 & 12.5 & 10.6 & 1.7 & 24.1 & 64 & 12.3 & 2.42 & 63.2 & 312 & $0.32^{\mathrm{b}}$ \\
\hline Clay loam & 467 & 6.8 & 2.1 & 13.6 & 8.3 & 2.3 & 20 & 166 & 7.6 & 2.97 & 21.3 & 631 & $0.38^{\mathrm{b}}$ \\
\hline Sandy clay & - & - & - & - & - & - & - & - & - & - & - & - & - \\
\hline Silty clay & 121 & 7.7 & 2.2 & 13.4 & 26.2 & 7.8 & 61.5 & 54 & 44.8 & 6.97 & 88.2 & 173 & $1.59^{\mathrm{b}}$ \\
\hline Clay & 333 & 14.6 & 1.7 & 39.5 & 354.3 & 1.3 & 1268.5 & 79 & 148.8 & 2.94 & 458.4 & 410 & $1.42^{\mathrm{b}}$ \\
\hline Silt & - & - & - & - & - & - & - & - & - & - & - & - & - \\
\hline Total & 4179 & 8.5 & 2.6 & 18.2 & 46 & 1.8 & 374.8 & 1895 & 41 & 3.4 & 174 & - & - \\
\hline
\end{tabular}

a The number soils included in calculation. ${ }^{b}$ ns: insignificant; ${ }^{c * *}$ : significant at $1 \%$ probability level. SD: standard deviation.

tion to properly estimate $K_{\text {sat }}$ and $S$. However, the SWIG database provides additional information such as land use, initial water content, and slope that might prove to be good predictors. A further analysis in this respect is however beyond the scope of this paper. More importantly, the present analysis in combination with the results provided in Table 11 shows that a texture-dominated derivation of $K_{\text {sat }}$ values, as implemented in most land surface models, does not provide adequate means to estimate $K_{\text {sat }}$.

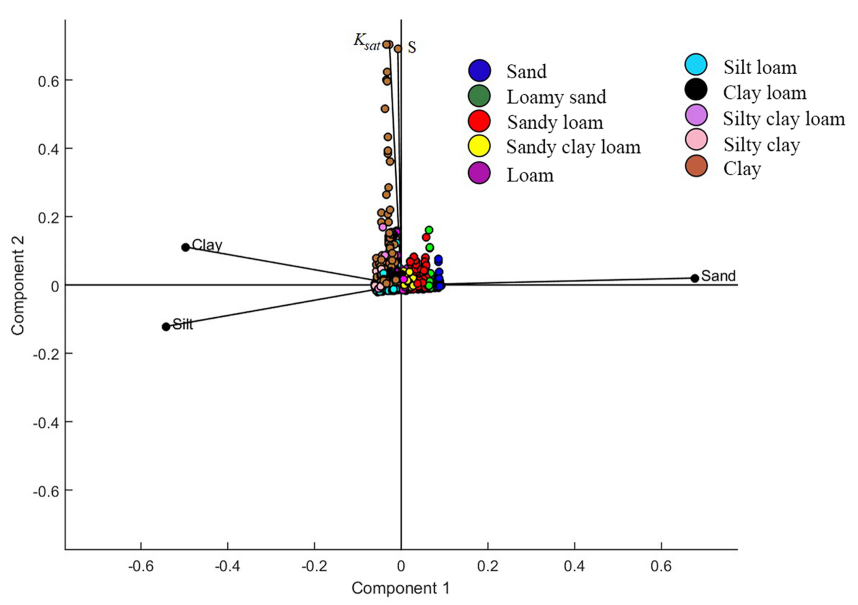

Figure 7. The relationships between clay, silt, sand contents and estimated hydraulic parameters ( $S$ and $K_{\text {sat }}$ ). 


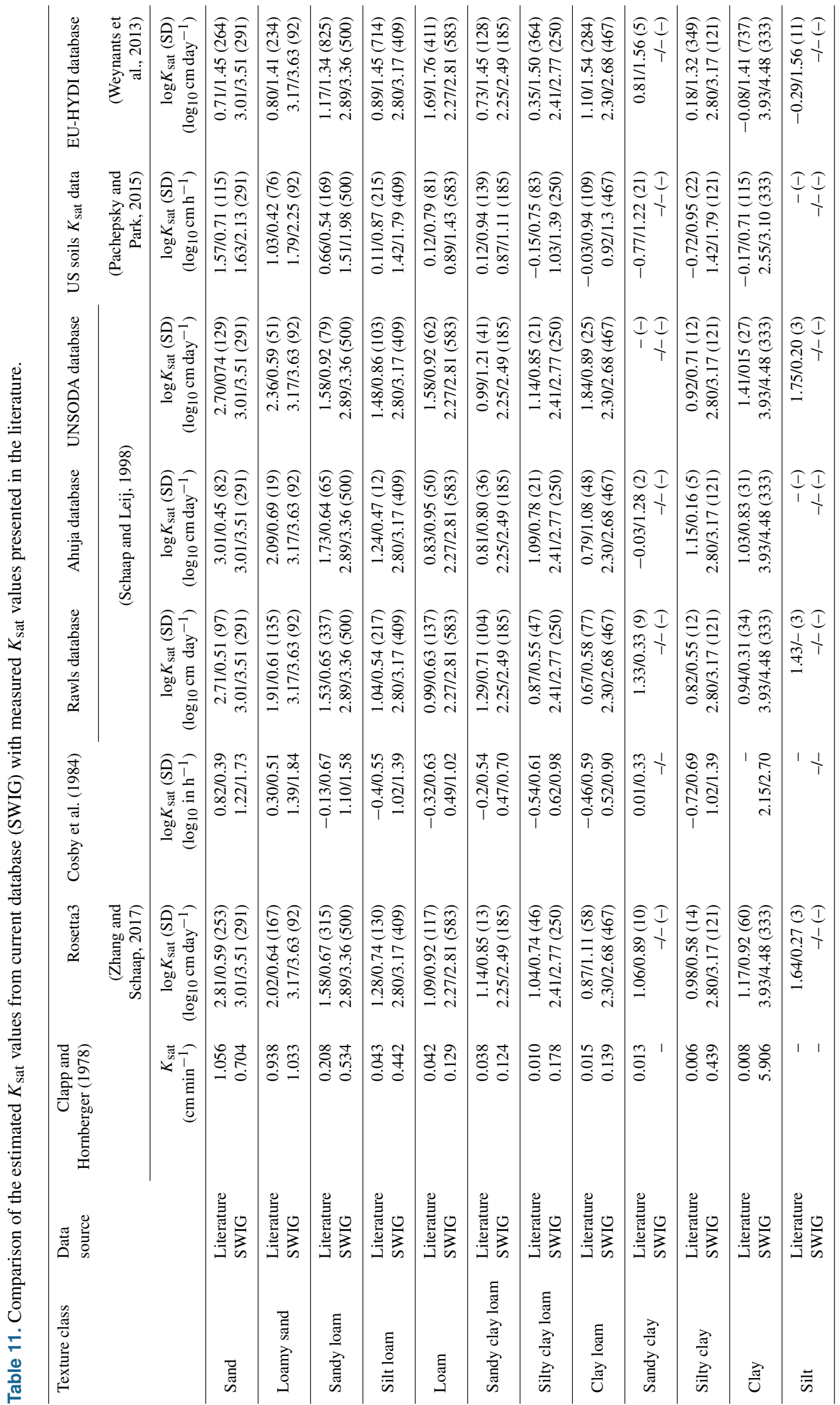




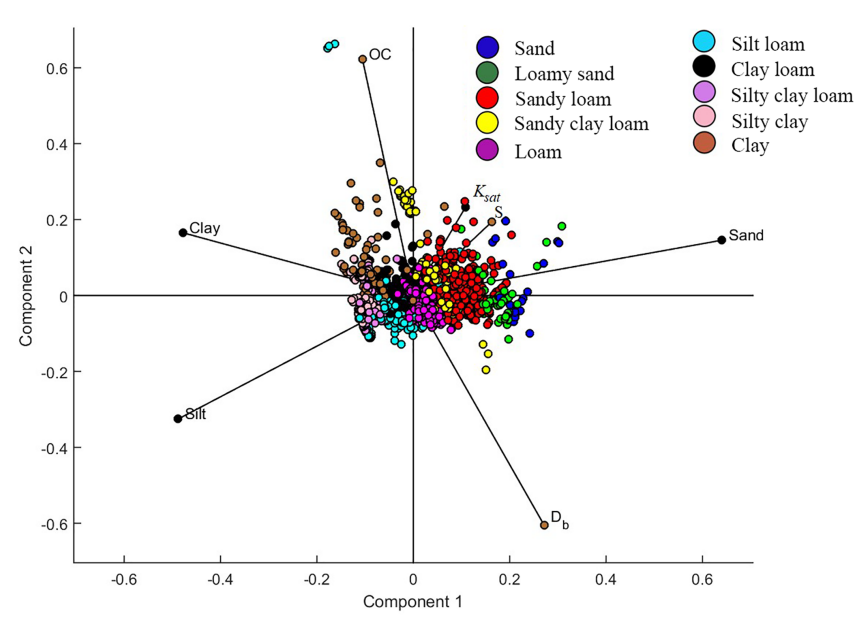

Figure 8. The relationships between clay, silt, sand contents, $D_{b}$, and OC and estimated hydraulic parameters ( $S$ and $\left.K_{\text {sat }}\right)$.

\subsection{Potential error and uncertainty in the SWIG database}

Similar to any other databases, the data presented in the SWIG database may be subject to different error sources and uncertainties. These include (1) transcription errors that occurred when implementing the measurement data into the EXCEL spreadsheets, (2) inaccuracy and uncertainties in determining related soil properties such as textural properties, (3) violation of the underlying assumption when performing the experiments, and (4) uncertainty (variability) in estimated soil hydraulic properties due to the different measurement methods. Unfortunately, none of these errors or uncertainty sources are under the control of the SWIG database authors, and quantification of these sources is often difficult, since the required information is often lacking. The uncertainty and variability related to the applied measurement techniques for estimated soil hydraulic properties may be assessed as information on the applied techniques is available; however, some of these methods may only have been used in few cases, making a statistical analysis difficult.

With respect to the transcription error, a strong effort has been made to double-check data transcription to prevent or at least to minimize any probable error of this nature. Values of soil properties such as textural composition are known to vary strongly between different laboratories and measurement methods. This is especially true for the finer textural classes like clay. Unfortunately, information on the measurement used to determine soil properties is mostly lacking or insufficient to assess the magnitude of errors or biases. Internationally, there are a number of standard methods used to measure soil properties and several methods may have been applied to measure the reported soil properties. In this regard, no conversion has been made and only raw data are reported in the database. However, we have supplied the references for all data (where available) that can be used to ascertain which methodologies were used, if so desired. Although supplying such information for each soil property may facilitate the use of the database, it would have required considerable additional work that could not be performed at this stage of development. Such additions could form the basis of a second version of the database that any readers should feel free to commence.

The uncertainty with respect to the effect of measurement techniques on quantifying the infiltration process itself may be analyzed from the SWIG database as it provides information on the type of measurement technique used. This analysis is again beyond the scope of this paper. Potential error and uncertainty sources with respect to the use of different measurements are discussed in the Supplement. The uncertainty of estimated soil hydraulic properties from infiltration measurements may be strongly controlled by the person performing the experiment but may also be due the different measurement windows of the methods in terms of measurement volume. The SWIG database provides information to quantify uncertainties introduced by difference in measurement volume and this analysis will be closely related to the assessment of the representative elementary volume, REV (see, for example, the work of Pachepsky on the scaling of saturated hydraulic conductivity).

Careful interpretation of the data, with respect to the details of the experimental and soil conditions, is also required when utilizing the SWIG database. For instance, the cases of soils coded 1211-1420 may at first seem odd, as they display very low infiltration rates for soils of a very high (>95\%) sand content; however, these unusual findings are explained by the soils being recorded as displaying water repellant characteristics. Another example is estimated values of $K_{\text {sat }}$ from clayey soils showing high values of $K_{\text {sat }}$ (e.g., soils coded 3746 to 3833 in the SWIG database). The $K_{\text {sat }}$ values for these soils were obtained using the single-ring infiltrometer method (Gonzalez-Sosa et al., 2010; Braud, 2015; Braud and Vandervaere, 2015) and were conducted in the field under ponded conditions, with vegetation cut but roots left in place. Macropores could have been activated, leading to an infiltration rate much higher than expected for clayey soils. There were also instances of very high values being obtained for forested land uses, and sometimes for grassland, which is probably explained by the visible cracks in the soil surface present in those cases

\subsection{Research potentials of the SWIG database}

We envision that the SWIG database offers a unique opportunity and information source to (1) evaluate infiltration methods and to assess their value in deriving soil hydraulic properties, (2) test different models and concepts for point-scale and grid-scale infiltration processes, (3) develop pedotransfer functions to estimate soil hydraulic properties such as the Mualem-van Genuchten parameters, (4) identify controls on infiltration processes, (5) validate global predictions of in- 
filtration from land surface models, (6) study more complex processes like preferential flow in soils, and (7) highlight the state-of-the-art understanding of the relationships between infiltration and several soil surface characteristics; for example, the SWIG database has already contributed to the scope of Morbidelli et al. (2018) to advance the knowledge of infiltration over sloping surfaces.

We are confident that the SWIG database is just a first step in collecting and archiving infiltration data and we expect that increasing amounts of data will become available in the near future. These data will be archived in the SWIG database and thus made available to the worldwide research community. In this regard, we are interested in receiving existing or newly measured infiltration curves and for this purpose the corresponding author will serve as point of contact or data can be made available through the International Soil Modeling Consortium, ISMC (https: //soil-modeling.org/, last access: 1 July 2018), for further archiving in the SWIG database.

\section{Data availability}

All collected data and related soil characteristics are provided online in *.xlsx and *.csv formats for reference and are available at https://doi.org/10.1594/PANGAEA.885492 (Rahmati et al., 2018). We add a disclaimer that the database is for public domain use only and can be copied freely by referencing it.

\section{Conclusion}

We have collected 5023 infiltration curves from field experiments from all over the world covering a broad range of soils, land uses, and climate regions. We estimated saturated hydraulic conductivity, $K_{\text {sat }}$, and sorptivity from more than 3000 infiltration curves and compared estimated $K_{\text {sat }}$ values with values from different databases published in the literature. We showed that contrary to the assumption made in many land surface and global climate models, texture is not the main controlling factor for $K_{\text {sat }}$. In addition, the variability of $K_{\text {sat }}$ derived from these field measurements is considerably larger than reported in the literature. The collected infiltration curves were archived as the SWIG database on the PANGAEA platform and are therefore available worldwide. The data are structured into *.xlsx and *.csv files and include metadata information for further use. Data analysis revealed that infiltration curves are lacking for clayey, sandy-textured, and stony soils. Also infiltration curve data are lacking for the northern and permafrost regions. Here, additional efforts are needed to collect more data as these regions are particularly sensitive to climate change, which will clearly affect the soil hydrology.
Supplement. The supplement related to this article is available online at: https://doi.org/10.5194/essd-10-1237-2018-supplement.

Author contributions. The idea of globally collecting soil infiltration data was put forward by MR and HV. Published data from literature were digitized by MR, LW, NM, and MK. Data contributors are MR, YAP, LM, SHS, HK, ZH, WAY, AAA, MZA, RAJ, ACDA, GA, RAA, HA, YB, JBA, BB, FB, GB, KB, IB, CC, AC, MC, RC, MC, BC, YC, WC, CC, APC, MBdO, JRdM, MFD, HE, IE, AF, AF, NF, MG, MHG, TAG, SG, EGH, RH, JJJ, DJ, SDK, HK, MKH, MKJ, LL, XL, MAL, LL, MVL, DM, DM, MSM, JDdOM, MRM, JM, FM, MHM, BPM, MPM, SM, RM, DMF, AAM, MRM, SBM, HM, KN, MRN, MVO, TBOF, MRPR, AP, SP, PEP, TP, MP, DJR, SR, MR, FPR, DR, JRC, OCRF, TS, HS, CS, RS, BS, MS, NS, ESM, MS, SS, RS, AAS, PS, ZS, RTM, ET, WGT, ARV, MV, TV, IV, JV, SW, TW, DY, MHY, SZ, YZe, YZh, and HZ. The data were collected by MR, HV, LW, and KVL. The data analysis was conceived, designed, and performed by MR, HV, LW, JV, SHS, CM, KVL, BT, FM, and RTM. The article was written by MR, HV, LW, LM, and HK. The article was actively revised several times by MR, LW, HV, YAP, MHY, SHS, MS, JP, ZH, AC, YC, LL, FM, RM, DMF, RS, WGT, HA, NS, RAA, IB, FPR, and SR. All authors checked the accuracy and/or commented on the contents of the paper.

Competing interests. The authors declare that they have no conflict of interest.

Acknowledgements. First author thanks the International and Scientific Cooperation Office of the University of Maragheh, Iran, as well as the research committee and board members of the university for their assistance in conducting the current work.

The financial support received from the Forschungszentrum Jülich $\mathrm{GmbH}$ is gratefully acknowledged by the first author.

Authors gratefully thank the International Soil Modeling Consortium (ISMC) and the International Soil Tillage Research Organization (ISTRO) for their help in distributing our call for data among researchers throughout the world.

Parts of data were gathered from the work that was supported by the UK-China Virtual Joint Centre for Improved Nitrogen Agronomy (CINAg, BB/N013468/1), which is jointly supported by the Newton Fund, via UK BBSRC and NERC.

The French Claduègne and Yzeron datasets were acquired during the ANR projects FloodScale (ANR-2011-BS56-027) and AVuUR (ANR-07-VULN-01), respectively.

Parts of the database were made available through research work carried out in the framework of LIFE+ projects funded by the EC.

The support of the Spanish Ministry of Economy through project CGL2014-53017-C2-1-R is acknowledged.

The support of the Czech Science Foundation through project no. 16-05665S is acknowledged.

The support of the Slovak Research and Development Agency through project no. APVV-15-0160 is acknowledged.

Authors are grateful to Atilla Nemes, Jan W. Hopmans, and Marnik Vanclooster for their time and attention in reviewing and commenting on this article. 
Edited by: David Carlson

Reviewed by: Marnik Vanclooster and Attila Nemes

\section{References}

Abagale, F. K., Abdulai, N., and Ojediran, J. O.: Effect of shea waste slurry on soil physical porperties in peri urban Tamale, Asian Journal of Science and Technology, 4, 36-41, 2012.

Abdallah, N. A., Wua, L. T., Widaa, A., and Elamin, M. B.: Rain infiltration into loess soil under different rain intensities and slope angles, International Journal of Scientific Engineering and Applied Science (IJSEAS), 2, 179-183, 2016.

Adindu Ruth, U., Igbokwe kelechi, K., Chigbu Timothy, O., and Ike-Amadi, C.: Application of Kostiakov's Infiltration Model on the Soils of Umudike, Abia State-Nigeria, American Journal of Environmental Engineering, 4, 1-6, https://doi.org/10.5923/j.ajee.20140401.01, 2014.

Alagna, V., Bagarello, V., Di Prima, S., Giordano, G., and Iovino, M.: Testing infiltration run effects on the estimated water transmission properties of a sandy-loam soil, Geoderma, 267, 24-33, https://doi.org/10.1016/j.geoderma.2015.12.029, 2016.

Al-Azawi, S. A.: Experimental evaluation of infiltration models, Journal of Hydrology (New Zealand), 24, 77-88, 1985.

Al-Ghazal, A. A.: Effect of tractor wheel compaction on bulk density and infiltration rate of a loamy sand soil in Saudi Arabia, Emir. J. Agric. Sci, 14, 24-33, https://doi.org/10.9755/ejfa.v13i1.4982, 2002.

Al-Kayssi, A. W. and Mustafa, S. H.: Modeling gypsifereous soil infiltration rate under different sprinkler application rates and successive irrigation events, Agr. Water Manage., 163, 66-74, https://doi.org/10.1016/j.agwat.2015.09.006, 2016.

Al Yamani, W., Green, S., Pangilinan, R., Dixon, S., and Clothier, B.: Sustainable irrigation of arid forests in Abu Dhabi using groundwater and treated sewage effluent, in: Integrated nutrient and water management for sustainable farming, edited by: Currie, L. D. and Singh, R., Occasional Report No. 29, Fertilizer and Lime Research Centre, Massey University, Palmerston North, New Zealand, 12 pp., 2016.

Angulo-Jaramillo, R., Vandervaere, J.-P., Roulier, S., Thony, J.L., Gaudet, J.-P., and Vauclin, M.: Field measurement of soil surface hydraulic properties by disc and ring infiltrometers: A review and recent developments, Soil Till. Res., 55, 1-29, https://doi.org/10.1016/S0167-1987(00)00098-2, 2000.

Angulo-Jaramillo, R., Bagarello, V., Iovino, M., and Lassabatère, L.: Infiltration Measurements for Soil Hydraulic Characterization, Springer International Publishing, New York, NY, USA, https://doi.org/10.1007/978-3-319-31788-5, 2016.

Arriaga, F. J., Kornecki, T. S., Balkcom, K. S., and Raper, R. L.: A method for automating data collection from a double-ring infiltrometer under falling head conditions, Soil Use Manage., 26, 61-67, https://doi.org/10.1111/j.1475-2743.2009.00249.x, 2010.

Arshad, E. I., Sarki, E. A., and Khan, E. Z. A.: Analysis of Water Transmission Behaviour in Sandy Loam Soil under Different Tillage Operations of Mould Board Plough applying/Using Different Infiltration Models, International Journal for Research in Applied Science \& Engineering Technology (IJRASET), 3, 254266, 2015.
Askari, M., Tanaka, T., Setiawan, B. I., and Saptomo, S. K.: Infiltration characteristics of tropical soil based on water retention data, Journal of Japan Society of Hydrology and Water Resources, 21, 215-227, 2008.

Ayu, I. W., Soemarno, S. P., and Java, I. I.: Assessment of Infiltration Rate under Different Drylands Types in Unter-Iwes Subdistrict Sumbawa Besar, Indonesia, Journal of Natural Sciences Research, 3, 7 pp., 2013.

Batlle-Aguilar, J., Schneider, S., Pessel, M., Tucholka, P., Coquet, Y., and Vachier, P.: Axisymetrical Infiltration in Soil Imaged by Noninvasive Electrical Resistivimetry, Soil Sci. Soc. Am. J., 73, 510-520, https://doi.org/10.2136/sssaj2007.0278, 2009.

Berglund, E. R., Ahyoud, A., and Tayaa, M. H.: Comparison of soil and infiltration properties of range and afforested sites in northern Morocco, Forest Ecol. Manag., 3, 295-306, https://doi.org/10.1016/0378-1127(80)90030-4, 1980.

Bertol, I., Barbosa, F. T., Bertol, C., and Luciano, R. V.: Water infiltration in two cultivated soils in Southern Brazil, Rev. Bras. Cienc. Solo, 39, 573-588, https://doi.org/10.1590/01000683rbcs20140304, 2015.

Bhardwaj, A. and Singh, R.: Development of a portable rainfall simulator infiltrometer for infiltration, runoff and erosion studies, Agr. Water Manage., 22, 235-248, https://doi.org/10.1016/03783774(92)90028-U, 1992.

Bhawan, J. V.: Infiltration studies in sher-umar river doab in narmada basin, National Institute of Hydrology, Jal Vigyan bhawan, India, 1997.

Biro, K., Pradhan, B., Buchroithner, M., and Makeschin, F.: The Effects of Different Land Use Types on Soil Compaction and Infiltration Rate in the Drylands Vertisol of Gadarif Region, Sudan, Tropentag 2010: World Food System - A Contribution from Europe, International Research on Food Security, Natural Resource Management and Rural Development, ETH Zurich, 1416 September 2010.

Braud, I.: Infiltration campaign Claduegne catchment, Ardeche, France, SEDOO OMP, https://doi.org/10.6096/mistralshymex.1321, 2015

Bowyer-Bower, T. A. S.: Effects of rainfall intensity and antecedent moisture on the steady-state infiltration rate in a semi-arid region, Soil Use Manage., 9, 69-75, https://doi.org/10.1111/j.14752743.1993.tb00932.x, 1993

Braud, I. and Vandervaere, J.: Analysis of infiltration tests performed in the Claduegne catchment in May-June 2012, contribution to WP3.4, "Documentation and mapping of soil hydraulic properties, soil geometry and vegetation cover of small catchments" of the FloodScale (2012-2015) ANR project, 66 pp., 2015.

Campbell, G. S.: Soil physics with BASIC: transport models for soil-plant systems, Elsevier, Amsterdam, the Netherlands, 1985.

Casanova, M. P.: Influence of slope gradient and aspect on soil hydraulic conductivity measured with tension infiltrometer, MSc thesis, Department of Soil Sciences Swedish University of Agricultural Sciences, Uppsala, 52 pp., 1998.

Chalhoub, M., Vachier, P., Coquet, Y., Darwish, T., Dever, L., and Mroueh, M.: Caractérisation des propriétés hydrodynamiques d'un sol de la Bekaa (Liban) sur les rives du fleuve Litani, Étude et Gestion des Sols, 16, 67-84, 2009.

Chartier, M., Rostagno, C., and Pazos, G.: Effects of soil degradation on infiltration rates in grazed semiarid rangelands of north- 
eastern Patagonia, Argentina, J. Arid Environ., 75, 656-661, https://doi.org/10.1016/j.jaridenv.2011.02.007, 2011.

Childs, E. C. and Collis-George, N.: The permeability of porous materials, P. Roy. Soc. Lond. A Mat., 201, 392-405, https://doi.org/10.1098/rspa.1950.0068, 1950.

Chow, V., Maidment, D., and Mays, L.: Applied hydrology, Editions McGraw-Hill, New York, 572 pp., 1988.

Cichota, R., Vogeler, I., Snow, V. O., and Webb, T. H.: Ensemble pedotransfer functions to derive hydraulic properties for New Zealand soils, Soil Res., 51, 94-111, https://doi.org/10.1071/sr12338, 2013.

Clapp, R. B. and Hornberger, G. M.: Empirical equations for some soil hydraulic properties, Water Resour. Res., 14, 601-604, https://doi.org/10.1029/WR014i004p00601, 1978.

Commandeur, P. R., Wass, E. F., Ministry of Forests and CanadaBritish Columbia Partnership Agreement on Forest Resource Development: FRDA II. (British Columbia), and Canadian Forest Service: Rainfall Simulation, Soil Infiltration and Surface Erosion on Skidroad Surfaces, Nelson Forest Region, Government of British Columbia, Canada, FRDA Report, Band 228, 28 pp., 1994.

Coquet, Y.: Etude in situ des phénomènes de retrait-gonflement des sols: application à deux sols tropicaux peu gonflants, ORSTOM, Paris, $355+119$ pp., Th. Sci. de la Terre: Pédol., Université d'Orléans, 1996.

Coquet, Y., Vachier, P., and Labat, C.: Vertical variation of nearsaturated hydraulic conductivity in three soil profiles, Geoderma, 126, 181-191, https://doi.org/10.1016/j.geoderma.2004.09.014, 2005.

Corradini, C., Morbidelli, R., and Govindaraju, R. S.: Infiltration Modeling, in: Handbook of Applied Hydrology, 2nd edn., edited by: Singh, V. P., McGraw-Hill, New York, 45-1-45-9, 2017.

Cosby, B., Hornberger, G., Clapp, R., and Ginn, T.: A statistical exploration of the relationships of soil moisture characteristics to the physical properties of soils, Water Resour. Res., 20, 682-690, https://doi.org/10.1029/WR020i006p00682, 1984.

Coutinho, A. P., Lassabatere, L., Montenegro, S., Antonino, A. C. D., Angulo-Jaramillo, R., and Cabral, J. J.: Hydraulic characterization and hydrological behaviour of a pilot permeable pavement in an urban centre, Brazil, Hydrol. Process., 30, 4242-4254, https://doi.org/10.1002/hyp.10985, 2016.

Dasgupta, S., Mohanty, B. P., and Köhne, J. M.: Soil Hydraulic Conductivities and their Spatial and Temporal Variations in a Vertisol, Soil Science Soc. Am. J., 70, 1872-1881, https://doi.org/10.2136/sssaj2006.0201, 2006.

Delage, P., Zadjaoui, A., Cui, Y. J., Ghabezloo, S., Pereira, J. M., and Tang, A. M.: Numerical modelling of infiltration profiles in the silt Tlemcen (Algeria), E3S Web of Conferences, 9, 11015, https://doi.org/10.1051/e3sconf/20160911015, 2016.

Di Prima, S., Lassabatere, L., Bagarello, V., Iovino, M., and Angulo-Jaramillo, R.: Testing a new automated single ring infiltrometer for Beerkan infiltration experiments, Geoderma, 262, 20-34, https://doi.org/10.1016/j.geoderma.2015.08.006, 2016.

Dušek, J., Lichner, L., Vogel, T., and Štekauerová, V.: Transport of iodide in structured soil under spring barley during irrigation experiment analyzed using dual-continuum model, Biologia, 68, 1094-1098 https://doi.org/10.2478/s11756-013-0249-4, 2013.

Fakher Nikche, A., Vafakhah, M., and Sadeghi, S. H. R.: Efficiency Evolution of Different Infiltration Models in Different Land Use and Soil Classes using Rainfall Simulator, Iranian Soil and Water Science, 24, 183-193, 2014.

Fakouri, T., Emami, H., and Ghahremani, B.: Estimation of cumulaive infiltration using particle size distribution in different agricultural landuses, Journal of Water Researches in Agriculture, 26, 379-390, 2011a.

Fakouri, T., Emami, H., and Ghahremani, B.: Effects of different landuses on soil water infiltration, Journal of Water Researches in Agriculture, 25, 195-206, 2011 b.

Fan, R., Zhang, X., Yang, X., Liang, A., Jia, S., and Chen, X.: Effects of tillage management on infiltration and preferential flow in a black soil, Northeast China, Chinese Geogr. Sci., 23, 312 320, https://doi.org/10.1007/s11769-013-0606-9, 2013.

Fodor, N., Sándor, R., Orfanus, T., Lichner, L., and Rajkai, K.: Evaluation method dependency of measured saturated hydraulic conductivity, Geoderma, 165, 60-68, https://doi.org/10.1016/j.geoderma.2011.07.004, 2011.

Gabriel, K. R.: The biplot graphic display of matrices with application to principal component analysis, Biometrika, 58, 453-467, https://doi.org/10.2307/2334381, 1971.

Gharaibeh, M. A., Ghezzehei, T. A., Albalasmeh, A. A., and Ma'in, Z. A.: Alteration of physical and chemical characteristics of clayey soils by irrigation with treated waste water, Geoderma, 276, 33-40, https://doi.org/10.1016/j.geoderma.2016.04.011, 2016.

Ghavimi Panah, M. H., Sadeghi, S. H. R., and Younesi, H.: Role of superficial biochar mulch produced from dairy factory waste on infiltration and runoff in small experimental plots, Iranian Journal of Soil and Water Research, 48, 905-916, 2017.

Giertz, S., Junge, B., and Diekkrüger, B.: Assessing the effects of land use change on soil physical properties and hydrological processes in the sub-humid tropical environment of West Africa, Phys. Chem. Earth, Pts. A/B/C, 30, 485-496, https://doi.org/10.1016/j.pce.2005.07.003, 2005.

Gonzalez-Sosa, E., Braud, I., Dehotin, J., Lassabatère, L., AnguloJaramillo, R., Lagouy, M., Branger, F., Jacqueminet, C., Kermadi, S., and Michel, K.: Impact of land use on the hydraulic properties of the topsoil in a small French catchment, Hydrol. Process., 24, 2382-2399, https://doi.org/10.1002/hyp.7640, 2010.

Green, W. H. and Ampt, G.: Studies on Soil Phyics, J. Agr. Sci., 4, 1-24, https://doi.org/10.1017/S0021859600001441, 2011.

Gupta, R., Rudra, R., Dickinson, W., and Wall, G.: Spatial and seasonal variations in hydraulic conductivity in relation to four determination techniques, Can. Water Resour. J., 19, 103-113, https://doi.org/10.4296/cwrj1902103, 1994.

Hatzigiannakis, E. and Panoras, A.: Report of results STU, physical, chemical and hydraulic properties of soil, Action 6: Assess land degradation caused by erosion in a pilot agricultural area, 433 pp., 2011.

Haverkamp, R., Ross, P. J., Smettem, K. R. J., and Parlange, J. Y.: Three-dimensional analysis of infiltration from the disc infiltrometer: 2. Physically based infiltration equation, Water Resour. Res., 30, 2931-2935, https://doi.org/10.1029/94WR01788, 1994.

Hazbavi, Z. and Sadeghi, S. H. R.: Potential effects of vinasse as a soil amendment to control runoff and soil loss, SOIL, 2, 71-78, https://doi.org/10.5194/soil-2-71-2016, 2016. 
Hengl, T., de Jesus, J. M., Heuvelink, G. B., Gonzalez, M. R., Kilibarda, M., Blagotić, A., Shangguan, W., Wright, M. N., Geng, X., Bauer-Marschallinger, B., Guevara, M. A., Vargas, R., MacMillan, R. A., Batjes, N. H., Leenaars, J. G. B., Ribeiro, E., Wheeler, I., Mantel, S., and Kempen, B.: SoilGrids250m: Global gridded soil information based on machine learning, PLoS ONE, 12, e0169748, https://doi.org/10.1371/journal.pone.0169748, 2017.

Hillel, D.: Introduction to environmental soil physics, Academic press, Massachusetts, USA, 2013.

Holzapfel, E. A., Mariño, M. A., Valenzuela, A., and Diaz, F.: Comparison of infiltration measuring methods for surface irrigation, J. Irrig. Drain. E.-ASCE, 114, 130-142, https://doi.org/10.1061/(ASCE)0733-9437(1988)114:1(130), 1988.

Hopmans, J. W., Parlange, J. Y., and Assouline, S.: Infiltration, in: The handbook of groundwater engineering, edited by: Delleur, J. W., CRC Press, Taylor \& Francis Group, Boca Raton, New York, USA, 2006.

$\mathrm{Hu}$, X., Li, Z.-C., Li, X.-Y., and Liu, L.-y.: Quantification of soil macropores under alpine vegetation using computed tomography in the Qinghai Lake Watershed, NE Qinghai-Tibet Plateau, Geoderma, 264, 244-251, https://doi.org/10.1016/j.geoderma.2015.11.001, 2016.

Huang, L., Zhang, P., Hu, Y., and Zhao, Y.: Vegetation succession and soil infiltration characteristics under different aged refuse dumps at the Heidaigou opencast coal mine, Global Ecology and Conservation, 4, 255-263, https://doi.org/10.1016/j.gecco.2015.07.006, 2015.

Huang, M., Barbour, S. L., Elshorbagy, A., Zettl, J. D., and Cheng $\mathrm{Si}, \mathrm{B}$. : Infiltration and drainage processes in multi-layered coarse soils, Can. J. Soil Sci., 91, 169-183, https://doi.org/10.4141/cjss09118, 2011.

Huwaldt, J. and Steinhorst, S: Plot Digitizer (Version 2.6. 8), Software, available at: https://sourceforge.net/projects/plotdigitizer/ files/ (last access: 5 July 2018), 2015.

Igbadun, H., Othman, M., and Ajayi, A.: Performance of Selected Water Infiltration Models in Sandy Clay Loam Soil in Samaru Zaria, Global Journal of Researches in Engineering: J General Engineering, 16, 8-14, 2016.

Ilstedt, U., Malmer, A., Verbeeten, E., and Murdiyarso, D.: The effect of afforestation on water infiltration in the tropics: a systematic review and meta-analysis, Forest Ecol. Manag., 251, 45-51, https://doi.org/10.1016/j.foreco.2007.06.014, 2007.

IUSS Working Group WRB: World reference base for soil resources 2006: a framework for international classification, correlation and communication, World soil resources reports No. 103. Rome: Food and Agriculture Organization of the United Nations, available at: http://www.fao.org/3/a-a0510e.pdf (last access: 24 June 2018), 2006.

Jačka, L., Pavlásek, J., Pech, P., and Kuráž, V.: Assessment of evaluation methods using infiltration data measured in heterogeneous mountain soils, Geoderma, 276, 74-83, https://doi.org/10.1016/j.geoderma.2016.04.023, 2016.

Jacques, D.: Analysis of water flow and solute transport at the field scale, $\mathrm{PhD}$ dissertation, Catholique University Leuven, Leuven, Belgium, 2000.

Jacques, D., Mohanty, B. P., and Feyen, J.: Comparison of alternative methods for deriving hydraulic properties and scaling factors from single-disc tension infiltrometer measurements, Water Resour. Res., 38, 25-1-25-14, https://doi.org/10.1029/2001WR000595, 2002.

Jemsi, S., Sayyad, G., Jafarnezhadi, A., and KashefiPour, S. M.: Development of infiltration rate pedotransfer functions using artificial neural networks and multiple linear regressions for Khuzestan province in south of Iran, International Journal of Agriculture: Research and Review, 3, 766-770, 2013.

Kashi, H., Emamgholizadeh, S., and Ghorbani, H.: Estimation of soil infiltration and cation exchange capacity based on multiple regression, ANN (RBF, MLP), and ANFIS models, Commun. Soil Sci. Plan., 45, 1195-1213, https://doi.org/10.1080/00103624.2013.874029, 2014.

Kavousi, S., Vafakhah, M., and Mahdian, M.: Evaluation of some infiltration models for different land uses in kojour watershed, Iranian of Irrigation \& Water Engineering, 4, 1-13, 2013.

Keesstra, S. D., Bouma, J., Wallinga, J., Tittonell, P., Smith, P., Cerdà, A., Montanarella, L., Quinton, J. N., Pachepsky, Y., van der Putten, W. H., Bardgett, R. D., Moolenaar, S., Mol, G., Jansen, B., and Fresco, L. O.: The significance of soils and soil science towards realization of the United Nations Sustainable Development Goals, SOIL, 2, 111-128, https://doi.org/10.5194/soil-2-111-2016, 2016.

Khan, G. S. and Strosser, P.: Soil survey of Pakistan, Director of Soil Servay of Punjab, Pakistan, 1-95, 1998.

Kheirfam, H., Sadeghi, S. H. R., Zarei Darki, B., and Homaee, M.: Controlling rainfall-induced soil loss from small experimental plots through inoculation of bacteria and cyanobacteria, Catena, 152, 40-46, https://doi.org/10.1016/j.catena.2017.01.006, 2017a.

Kheirfam, H., Sadeghi, S. H. R., Homaee, M., and Zarei Darki, B.: Quality improvement of an erosion-prone soil through microbial enrichment, Soil Till. Res., 165, 230-238, https://doi.org/10.1016/j.still.2016.08.021, 2017b.

Kiani-Harchegani, M., Sadeghi, S. H. R., and Asadi, H.: Comparing grain size distribution of sediment and original soil under raindrop detachment and raindrop-induced and flow transport mechanism, Hydrolog. Sci. J., 63, 312-323, https://doi.org/10.1080/02626667.2017.1414218, 2018.

Klute, A.: Some theoretical aspects of the flow of water in unsaturated soils, Soil Sci. Soc. Am. J., 16, 144-148, https://doi.org/10.2136/sssaj1952.03615995001600020008x, 1952.

Kottek, M., Grieser, J., Beck, C., Rudolf, B., and Rubel, F.: World map of the Köppen-Geiger climate classification updated, Meteorol. Z., 15, 259-263, https://doi.org/10.1127/09412948/2006/0130, 2006

Kutílek, M. and Krejča, M.: Three-parameter infiltration equation of Philip type, Vodohosp. Čas., 35, 52-61, 1987.

Lal, R. and Shukla, M. K.: Principles of soil physics, CRC Press, 2004.

Lassabatere, L., Angulo-Jaramillo, R., Goutaland, D., Letellier, L., Gaudet, J., Winiarski, T., and Delolme, C.: Effect of the settlement of sediments on water infiltration in two urban infiltration basins, Geoderma, 156, 316-325, https://doi.org/10.1016/j.geoderma.2010.02.031, 2010.

Lassabatere, L., Yilmaz, D., Peyrard, X., Peyneau, P. E., Lenoir, T., Šimůnek, J., and Angulo-Jaramillo, R.: New analytical model for cumulative infiltration into dual-permeability soils, Va- 
dose Zone J., 13, 1-15, https://doi.org/10.2136/vzj2013.10.0181, 2014.

Latorre, B., Peña, C., Lassabatere, L., Angulo-Jaramillo, R., and Moret-Fernández, D.: Estimate of soil hydraulic properties from disc infiltrometer three-dimensional infiltration curve. $\mathrm{Nu}$ merical analysis and field application, J. Hydrol., 527, 1-12, https://doi.org/10.1016/j.jhydrol.2015.04.015, 2015.

Li, X.-Y., Zhang, S.-Y., Peng, H.-Y., Hu, X., and Ma, Y.-J.: Soil water and temperature dynamics in shrub-encroached grasslands and climatic implications: results from Inner Mongolia steppe ecosystem of north China, Agr. Forest Meteorol., 171, 20-30, https://doi.org/10.1016/j.agrformet.2012.11.001, 2013.

Lichner, L., Eldridge, D., Schacht, K., Zhukova, N., Holko, L., Sir, M., and Pecho, J.: Grass cover influences hydrophysical parameters and heterogeneity of water flow in a sandy soil, Pedosphere, 21, 719-729, https://doi.org/10.1016/S1002-0160(11)60175-6, 2011.

Lichner, L., Holko, L., Zhukova, N., Schacht, K., Rajkai, K., Fodor, N., and Sándor, R.: Plants and biological soil crust influence the hydrophysical parameters and water flow in an aeolian sandy soil, J. Hydrol. Hydromech., 60, 309-318, https://doi.org/10.2478/v10098-012-0027-y, 2012.

Lichner, L., Hallett, P. D., Drongová, Z., Czachor, H., Kovacik, L., Mataix-Solera, J., and Homolák, M.: Algae influence the hydrophysical parameters of a sandy soil, Catena, 108, 58-68, https://doi.org/10.1016/j.catena.2012.02.016, 2013.

Liebig, M., Tanaka, D., and Wienhold, B. J.: Tillage and cropping effects on soil quality indicators in the northern Great Plains, Soil Till. Res., 78, 131-141, https://doi.org/10.1016/j.still.2004.02.002, 2004.

Lipiec, J., Kuś, J., Słowińska-Jurkiewicz, A., and Nosalewicz, A.: Soil porosity and water infiltration as influenced by tillage methods, Soil Till. Res., 89, 210-220, https://doi.org/10.1016/j.still.2005.07.012, 2006.

Ma, D., Zhang, J., Horton, R., Wang, Q., and Lai, J.: Analytical method to determine soil hydraulic properties from vertical infiltration experiments, Soil Sci. Soc. Am. J., 81, 1303-1314, https://doi.org/10.2136/sssaj2017.02.0061, 2017.

Machiwal, D., Jha, M. K., and Mal, B. C.: Modelling infiltration and quantifying spatial soil variability in a wasteland of Kharagpur, India, Biosyst. Eng., 95, 569-582, https://doi.org/10.1016/j.biosystemseng.2006.08.007, 2006.

Machiwal, D., Dayal, D., and Kumar, S.: Estimating water balance of small reservoirs in arid regions: a case study from Kachchh, India, Agr. Res., 6, 57-65, https://doi.org/10.1007/s40003-0160243-5, 2017

Mallmann, M. S.: Water infiltration in soil conditioned by use of cover crops, MSc thesis, Soil Science, Federal University of Santa Maria, Santa Maria, 72 pp., 2017.

Mao, L., Bralts, V. F., Pan, Y., Liu, H., and Lei, T.: Methods for measuring soil infiltration: State of the art, In. J. Agr. Biol. Eng., 1, 22-30, https://doi.org/10.3965/j.issn.1934-6344.2008.01.022030, 2008a.

Mao, L., Lei, T., Li, X., Liu, H., Huang, X., and Zhang, Y.: A linear source method for soil infiltrability measurement and model representations, J. Hydrol., 353, 49-58, https://doi.org/10.1016/j.jhydrol.2008.01.009, 2008b.

Mao, L., Li, Y., Hao, W., Zhou, X., Xu, C., and Lei, T.: A new method to estimate soil water infiltration based on a modified Green-Ampt model, Soil Till. Res., 161, 31-37, https://doi.org/10.1016/j.still.2016.03.003, 2016.

Matula, S.: The influence of tillage treatments on water infiltration into soil profile, Plant Soil Environ., 49, 298-306, 2003.

McKenzie, N., Coughlan, K., and Cresswell, H.: Soil physical measurement and interpretation for land evaluation, Csiro Publishing, Australia, 2002.

Medinski, T., Mills, A., and Fey, M.: Infiltrability in soils from south-western Africa: effects of texture, electrical conductivity and exchangeable sodium percentage, South African Journal of Plant and Soil, 26, 157-163, https://doi.org/10.1080/02571862.2009.10639949, 2009.

Mertens, J., Jacques, D., Vanderborght, J., and Feyen, J.: Characterisation of the field-saturated hydraulic conductivity on a hillslope: in situ single ring pressure infiltrometer measurements, J. Hydrol., 263, 217-229, https://doi.org/10.1016/S00221694(02)00052-5, 2002.

Mertens, J., Madsen, H., Feyen, L., Jacques, D., and Feyen, J.: Including prior information in the estimation of effective soil parameters in unsaturated zone modelling, J. Hydrol., 294, 251269, https://doi.org/10.1016/j.jhydrol.2004.02.011, 2004.

Mertens, J., Madsen, H., Kristensen, M., Jacques, D., and Feyen, J.: Sensitivity of soil parameters in unsaturated zone modelling and the relation between effective, laboratory andin situ estimates, Hydrol. Process., 19, 1611-1633, https://doi.org/10.1002/hyp.5591, 2005.

Miller, K. A., Elliott, J., and Friday, N.: Soils Infiltration Data for Selected Wyoming Watersheds, 1998-1999: Cheyenne, Wyoming, U.S. Geological Survey Open-File Report 2005-1322, 74 pp., 2005.

Mohammed, A. M. E., Mohamed, H. I., and Elramlawi, H. R.: Comparison of infiltration measuring techniques for furrow irrigation in cracking clay soil, J. Sci. Technol., 8, 1-7, 2007

Mohanty, B., Kanwar, R. S., and Everts, C.: Comparison of saturated hydraulic conductivity measurement methods for a glacial-till soil, Soil Sci. Soc. Am. J., 58, 672-677, https://doi.org/10.2136/sssaj1994.03615995005800030006x, 1994.

Morbidelli, R., Corradini, C., Saltalippi, C., Flammini, A., and Rossi, E.: Infiltration-soil moisture redistribution under natural conditions: experimental evidence as a guideline for realizing simulation models, Hydrol. Earth Syst. Sci., 15, 2937-2945, https://doi.org/10.5194/hess-15-2937-2011, 2011.

Morbidelli, R., Saltalippi, C., Flammini, A., Cifrodelli, M., Picciafuoco, T., Corradini, C., and Govindaraju, R. S.: In situ measurements of soil saturated hydraulic conductivity: Assessment of reliability through rainfall-runoff experiments, Hydrol. Process., 31, 3084-3094, https://doi.org/10.1002/hyp.11247, 2017.

Morbidelli, R., Saltalippi, C., Flammini, A., and Govindaraju, R. S.: Role of slope on infiltration: a review, J. Hydrol., 557, 878-886, https://doi.org/10.1016/j.jhydrol.2018.01.019, 2018.

Murray, C. D. and Buttle, J. M.: Infiltration and soil water mixing on forested and harvested slopes during spring snowmelt, Turkey Lakes Watershed, central Ontario, J. Hydrol., 306, 1-20, https://doi.org/10.1016/j.jhydrol.2004.08.032, 2005.

Naeth, M. A., Chanasyk, D. S., and Bailey, A. W.: Applicability of the Kostiakov equation to mixed prairie and fescue grasslands of Alberta, J. Range Manage., 44, 18-21, https://doi.org/10.2307/4002631, 1991. 
Návar, J. and Synnott, T. J.: Soil infiltration and land use in Linares, NL, Mexico, Terra Latinoamericana, 18, 255-262, 2000.

Nikghalpour, M., Asadi, H., and Gorji, M.: Evaluation of spatial distribution of water infiltration rate and its relation with some physical and chemical properties in the Kuhin region, Journal of Soil Researches (Soil and Water Science), 30, 201-213, 2016.

Ogbe, V., Jayeoba, O., and Ode, S.: Comparison of Four Soil Infiltration Models on A Sandy Soil in Lafia. Southern Guinea Savanna Zone of Nigeria, Production Agriculture and Technology (PAT), 7, 116-126, 2011.

Ojha, S., Machiwal, D., and Purohit, R. C.: Infiltration modeling in submergence area of a water harvesting structure: a case study, Indian Journal of Soil Conservation, 41, 8-13, 2013.

Oliveira, M. B.: Performance analysis of infiltration equations and of methods of determination of field capacity for soils at a watershed in the São José de Ubá county (state of Rio de Janeiro, Brazil), MSc thesis, Civil Engineering Program, Alberto Luiz Coimbra Institute for Postgraduate Studies and Research in Engineering, Federal University of Rio de Janeiro, COPPE/UFRJ, Rio de Janeiro, RJ, Brasil, 2005.

Omuto, T. C., Minasny, B., McBratney, A. B., and Biamah, E. K.: Nonlinear mixed effect modelling for improved estimation of water retention and infiltration parameters, J. Hydrol., 330, 748758, https://doi.org/10.1016/j.jhydrol.2006.05.006, 2006.

Othman, M. and Ajayi, A.: Infiltration Characteristics of Organic Amended Soils, Global Journal of Research in Engineering, 16, 35-39, 2016.

Pachepsky, Y. and Park, Y.: Saturated hydraulic conductivity of US soils grouped according to textural class and bulk density, Soil Sci. Soc. Am. J., 79, 1094-1100, https://doi.org/10.2136/sssaj2015.02.0067, 2015.

Pahlavan-Rad, M. R.: Study on spatial variability of soil infiltration and saturated hydraulic conductivity in the lands of Sistan plain using geostatistical and random forest methods, Annual report, Sistan Agricultural and Natural Resources Research and Education Center, Zabol, Areeo, Iran, 2017.

Parchami-Araghi, F., Mirlatifi, S. M., Ghorbani Dashtaki, S., and Mahdian, M. H.: Point estimation of soil water infiltration process using Artificial Neural Networks for some calcareous soils, J. Hydrol., 481, 35-47, https://doi.org/10.1016/j.jhydrol.2012.12.007, 2013.

Perkins, S. R. and McDaniel, K. C.: Infiltration and Sediment Rates Following Creosotebush Control With Tebuthiuron, Rangeland Ecol. Manag., 58, 605-613, https://doi.org/10.2111/05-048r1.1, 2005.

Philip, J.-R.: The theory of infiltration: 1 . The infiltration equation and its solution, Soil Sci., 83, 345-358, 1957.

Poesen, J.: Soil erosion in the Anthropocene: Research needs, Earth Surf. Proc. Land., 43, 64-84, https://doi.org/10.1002/esp.4250, 2018.

Pulido Moncada, M., Helwig Penning, L., Timm, L. C., Gabriels, D., and Cornelis, W. M.: Visual examinations and soil physical and hydraulic properties for assessing soil structural quality of soils with contrasting textures and land uses, Soil Till. Res., 140, 20-28, https://doi.org/10.1016/j.still.2014.02.009, 2014.

Qi, D. H. and Liu, Z. Q.: Soil Infiltration Characteristics under Different Land at Western Yunnan Plateau, Adv. Mat. Res., 694, 884-885, 2014.
Qian, F., Cheng, D., and Liu, J.: Analysis of the Water and Soil Erosion and Infiltration Characteristic in Ziquejie Terrace, IERI Procedia, 9, 13-19, https://doi.org/10.1016/j.ieri.2014.09.034, 2014.

Quadri, M., Angulo-Jaramillo, R., Vauclin, M., Clothier, B., and Green, S.: Axisymmetric transport of water and solute underneath a disk permeameter: Experiments and numerical model, Soil Sci. Soc. Am. J., 58, 696-703, https://doi.org/10.2136/sssaj1994.03615995005800030009x, 1994.

Rahmati, M.: Reliable and accurate point-based prediction of cumulative infiltration using soil readily available characteristics: a comparison between GMDH, ANN, and MLR, J. Hydrol., 551, 81-91, https://doi.org/10.1016/j.jhydrol.2017.05.046, 2017.

Rahmati, M., Weihermüller, L., Vereecken, H.: Soil Water Infiltration Global (SWIG) Database, Supplement to: Rahmati, M et al. (2018): Development and Analysis of Soil Water Infiltration Global Database, in Earth System Science Data, PANGAEA, https://doi.org/10.1594/PANGAEA.885492, 2018.

Rawls, W., Yates, P., and Asmussen, L.: Calibration of selected infiltration equations for the Georgia Coastal Plain, Agricultural Research Service, U.S. Dept. of Agriculture, New Orleans, Report ARS-S-113, 110 pp., 1976.

Rei, I., Kazufumi, I., Yuki, I., Tatsuro, S., and Yukihiro, S.: Evaluation of Infiltration Capacity and Water Retention Potential of Amended Soil Using Bamboo Charcoal and Humus for Urban Flood Prevention, J. Earth Sci. Eng., 6, 150-163, https://doi.org/10.17265/2159-581x/2016.03.002, 2016.

Rezaei, M., Seay, T., Seuntjens, P., Joris, I., Boënne, W., Van Meirvenne, M., and Cornelis, W.: Predicting saturated hydraulic conductivity in a sandy grassland using proximally sensed apparent electrical conductivity, J. Appl. Geophys., 126, 35-41, https://doi.org/10.1016/j.jappgeo.2016.01.010, 2016a.

Rezaei, M., Seuntjens, P., Shahidi, R., Joris, I., Boënne, W., Al-Barri, B., and Cornelis, W.: The relevance of in-situ and laboratory characterization of sandy soil hydraulic properties for soil water simulations, J. Hydrol., 534, 251-265, https://doi.org/10.1016/j.jhydrol.2015.12.062, 2016 b.

Richards, L. A.: Capillary conduction of liquids through porous mediums, J. Appl. Phys., 1, 318-333, https://doi.org/10.1063/1.1745010, 1931.

Robinson, D. A., Jones, S. B., Lebron, I., Reinsch, S., Domínguez, M. T., Smith, A. R., Jones, D. L., Marshall, M. R., and Emmett, B. A.: Experimental evidence for drought induced alternative stable states of soil moisture, Scientific reports, 6, 20018, https://doi.org/10.1038/srep20018, 2016.

Robinson, D., Lebron, I., Smith, A., Marshall, M., Emmett, B., Reinsch, S., Cooper, D., and Brooks, M.: Soil hydraulic property data from the Climoor fieldsite in the Clocaenog Forest (20102012), NERC Environmental Information Data Centre, 2017.

Rodrigo-Comino, J., Sinoga, J. R., González, J. S., GuerraMerchán, A., Seeger, M., and Ries, J.: High variability of soil erosion and hydrological processes in Mediterranean hillslope vineyards (Montes de Málaga, Spain), Catena, 145, 274-284, https://doi.org/10.1016/j.catena.2016.06.012, 2016.

Rodrigo-Comino, J., Taguas, E., Seeger, M., and Ries, J. B.: Quantification of soil and water losses in an extensive olive orchard catchment in Southern Spain, J. Hydrol., 556, 749-758, https://doi.org/10.1016/j.jhydrol.2017.12.014, 2018. 
Rubel, F., Brugger, K., Haslinger, K., and Auer, I.: The climate of the European Alps: Shift of very high resolution KöppenGeiger climate zones 1800-2100, Meteorol. Z., 26, 115-125, https://doi.org/10.1127/metz/2016/0816, 2017.

Ruprecht, J. and Schofield, N.: Infiltration characteristics of a complex lateritic soil profile, Hydrol. Process., 7, 87-97, https://doi.org/10.1002/hyp.3360070109, 1993.

Sadeghi, S. H. R., Hazbavi, Z., and Younesi, H.: Sustainable watershed management through applying appropriate level of soil amendments, 2nd International Conference on Sustainable Watershed Management, CRC Press, SUWAMA2014, Sarigerme, Turkey, 13-15 October 2014, 183-185, 2014.

Sadeghi, S. H. R., Moghaddam, E., and Khaledi Darvishan, A.: Effects of subsequent rainfall events on runoff and soil erosion components from small plots treated by vinasse, Catena, 138, 112, https://doi.org/10.1016/j.catena.2015.11.007, 2016a.

Sadeghi, S. H. R., Hazbavi, Z., and Kiani-Harchegani, M.: Controllability of runoff and soil loss from small plots treated by vinasse-produced biochar, Sci. Total Environ., 541, 483-490, https://doi.org/10.1016/j.scitotenv.2015.09.068, $2016 \mathrm{~b}$.

Sadeghi, S. H. R., Hazbavi, Z., Younesi, H., and Bahramifar, N.: Trade-off between runoff and sediments from treated erosion plots and polyacrylamide and acrylamide residues, Catena, 142, 213-220, https://doi.org/10.1016/j.catena.2016.03.013, 2016 c.

Sadeghi, S. H. R., Kheirfam, H., Homaee, M., Zarei Darki, B., and Vafakhah, M.: Improving runoff behavior resulting from direct inoculation of soil micro-organisms, Soil Till. Res., 171, 35-41, https://doi.org/10.1016/j.still.2017.04.007, 2017a.

Sadeghi, S. H. R., Kiani-Harchegani, M., and Asadi, H.: Variability of particle size distributions of upward/downward splashed materials in different rainfall intensities and slopes, Geoderma, 290, 100-106, https://doi.org/10.1016/j.geoderma.2016.12.007, $2017 b$.

Sahoo, A. K., Dirmeyer, P. A., Houser, P. R., and Kafatos, M.: A study of land surface processes using land surface models over the Little River Experimental Watershed, Georgia, J. Geophys. Res., 113, D20121, https://doi.org/10.1029/2007JD009671, 2008.

Saito, T., Yasuda, H., Suganuma, H., Inosako, K., Abe, Y., and Kojima, T.: Predicting Soil Infiltration and Horizon Thickness for a Large-Scale Water Balance Model in an Arid Environment, Water, 8, 96, https://doi.org/10.3390/w8030096, 2016.

Sándor, R., Lichner, L., Filep, T., Balog, K., Lehoczky, É., and Fodor, N.: Spatial variability of hydrophysical properties of fallow sandy soils, Biologia, 70, 1468-1473, https://doi.org/10.1515/biolog-2015-0182, 2015.

Sarmadian, F. and Taghizadeh-Mehrjardi, R.: Estimation of infiltration rate and deep percolation water using feed-forward neural networks in Gorgan Province, Eurasian Journal of Soil Science, 3, 1-6, https://doi.org/10.18393/ejss.03148, 2014.

Sauwa, M. M., Chiroma, A. M., Waniyo, U. U., Ngala, A. L., and Danmowa, N. M.: Water transmission properties of a sandy loam soil under different tillage practices in Maiduguri, Nigeria, Agriculture and Biology Journal of North America, 4, 227-251, https://doi.org/10.5251/abjna.2013.4.3.227.233, 2013.

Schaap, M. G. and Leij, F. J.: Database-related accuracy and uncertainty of pedotransfer functions, Soil Sci., 163, 765-779, https://doi.org/10.1097/00010694-199810000-00001, 1998.
Scotter, D., Clothier, B., and Sauer, T.: A critical assessment of the role of measured hydraulic properties in the simulation of absorption, infiltration and redistribution of soil water, Agr. Water Manage., 15, 73-86, https://doi.org/10.1016/0378-3774(88)90144-8, 1988.

Sepehrnia, N., Hajabbasi, M. A., Afyuni, M., and Lichner, L.: Extent and persistence of water repellency in two Iranian soils, Biologia, 71, 1137-1143, https://doi.org/10.1515/biolog-20160135, 2016.

Sepehrnia, N., Hajabbasi, M. A., Afyuni, M., and Lichner, L.: Soil water repellency changes with depth and relationship to physical properties within wettable and repellent soil profiles, J. Hydrol. Hydromech., 65, 99-104, https://doi.org/10.1515/johh2016-0055, 2017.

Sharifi Moghaddam, E., Sadeghi, S. H. R., and Khaledi Darvishan, A.: Small plot soil hydrologic components as affected by application of vinasse organicresidue, Iranian Journal of Soil and Water Research, 45, 499-508, 2014.

Shirazi, M. A. and Boersma, L.: A unifying quantitative analysis of soil texture, Soil Sci. Soc. Am. J., 48, 142-147, https://doi.org/10.2136/sssaj1984.03615995004800010026x, 1984.

Shukla, M., Lal, R., and Ebinger, M.: Tillage effects on physical and hydrological properties of a typic Argiaquoll in central Ohio, Soil Sci., 168, 802-811, https://doi.org/10.1097/01.ss.0000100470.96182.4a, 2003.

Shukla, M., Lal, R., Ebinger, M., and Meyer, C.: Physical and chemical properties of soils under some piñon-juniper-oak canopies in a semi-arid ecosystem in New Mexico, J. Arid Environ., 66, 673685, https://doi.org/10.1016/j.jaridenv.2005.12.002, 2006.

Sihag, P., Tiwari, N., and Ranjan, S.: Estimation and intercomparison of infiltration models, Water Sci., 31, 34-43, https://doi.org/10.1016/j.wsj.2017.03.001, 2017.

Smith, R. E. and Parlange, J. Y.: A parameter-efficient hydrologic infiltration model, Water Resour. Res., 14, 533-538, https://doi.org/10.1029/WR014i003p00533, 1978.

Smith, R. E., Smettem, K. R., and Broadbridge, P.: Infiltration theory for hydrologic applications, American Geophysical Union, 2002.

Sorman, A. U., Abdulrazzak, M. J., and Ugas, M. A. S.: Application of infiltration models to field data from Wadi Tabalah, Saudi Arabia, Application of Tracers in Arid Zone Hydrology, 232, 305316, 1995.

Su, L., Wang, Q., Shan, Y., and Zhou, B.: Estimating Soil Saturated Hydraulic Conductivity using the Kostiakov and Philip Infiltration Equations, Soil Sci. Soc. Am. J., 80, 1463-1475, https://doi.org/10.2136/sssaj2016.04.0125, 2016.

Sukhanovskij, Y. P., Vytovtov, V. A., Prushchik, A. V., Solov'eva, Y. A., and Sanzharova, S. I.: Assessment of soil infiltration capacity by using portable rainfall simulator, Byulleten Pochvennogo instituta im. V.V. Dokuchaeva, 78, 26-35, 2015.

Suzuki, K.: Estimation of Snowmelt Infiltration into Frozen Ground and Snowmelt Runoff in the Mogot Experimental Watershed in East Siberia, International Journal of Geosciences, 4, 13461354, https://doi.org/10.4236/ijg.2013.410131, 2013.

Teague, N. F.: Near surface infiltration measurements and the implications for artificial recharge, MSc thesis, Geological Sciences, San Diego State University, San Diego, 2010. 
Teixeira, W. G., Schroth, G., Marques, J. D., and Huwe, B.: Unsaturated Soil Hydraulic Conductivity in the Central Amazon: Field Evaluations, in: Application of Soil Physics in Environmental Analyses, edited by: Teixeira, W., Ceddia, M., Ottoni, M., and Donnagema, G., Progress in Soil Science, Springer, Cham, 283305, https://doi.org/10.1007/978-3-319-06013-2_13, 2014.

Thierfelder, C. and Wall, P. C.: Effects of conservation agriculture techniques on infiltration and soil water content in Zambia and Zimbabwe, Soil Till. Res., 105, 217-227, https://doi.org/10.1016/j.still.2009.07.007, 2009.

Thierfelder, C., Stahr, K., and Edgar, A. C.: Soil crusting and sealing in the Andean Hillsides of Colombia and its impact on water infiltration, in: Technological and Institutional Innovations for Sustainable Rural Development: Deutscher Tropentag 2003: International research on food security, natural resource management and rural development: Book of abstracts, Georg-AugustUniversität Göttingen, 8-10 October 2003, edited by: Wollny, C., Deininger, A., Bhandari, N., Maass, B., Manig, W., Muuss, U., Brodbeck, F., and Howe, I., klartext GmbH, Göttingen, Germany, 1-10, 2003.

Thierfelder, C., Chivenge, P., Mupangwa, W., Rosenstock, T. S., Lamanna, C., and Eyre, J. X.: How climate-smart is conservation agriculture (CA)? - its potential to deliver on adaptation, mitigation and productivity on smallholder farms in southern Africa, Food Secur., 9, 537-560, https://doi.org/10.1007/s12571017-0665-3, 2017.

Uloma, A., Onyekachi, C., Torti, E., and Amos, U.: Infiltration characteristics of soils of some selected schools in aba, nigeria, Archives of Applied Science Research, 5, 11-15, 2013.

US Department of Agriculture (USDA): Keys to Soil Taxonomy, 12th edn., U.S. Government Printing Office, available at: https://www.nrcs.usda.gov/wps/portal/nrcs/detail/soils/ survey/class/taxonomy/?cid=nrcs142p2_053580 (last access: 24 June 2018), 2014.

van der Kamp, G., Hayashi, M., and Gallén, D.: Comparing the hydrology of grassed and cultivated catchments in the semi-arid Canadian prairies, Hydrol. Process., 17, 559-575, https://doi.org/10.1002/hyp.1157, 2003.

Van Looy, K., Bouma, J., Herbst, M., Koestel, J., Minasny, B., Mishra, U., Montzka, C., Nemes, A., Pachepsky, Y., and Padarian, J.: Pedotransfer functions in Earth system science: challenges and perspectives, Rev. Geophys., 55, 1199-1256, https://doi.org/10.1002/2017RG000581, 2017.

Vogel, T. and Cislerova, M.: A scaling-based interpretation of a field infiltration experiment, J. Hydrol., 142, 337-347, https://doi.org/10.1016/0022-1694(93)90017-4, 1993.

Vogeler, I., Cichota, R., Sivakumaran, S., Deurer, M., and McIvor, I.: Soil assessment of apple orchards under conventional and organic management, Soil Res., 44, 745-752, https://doi.org/10.1071/SR06096, 2006.

Votrubova, J., Dohnal, M., Vogel, T., Tesar, M., Jelinkova, V., and Cislerova, M.: Ponded infiltration in a grid of permanent singlering infiltrometers: Spatial versus temporal variability, J. Hydrol. Hydrom., 65, 244-253, https://doi.org/10.1515/johh-2017-0015, 2017.

Wang, G., Fang, Q., Wu, B., Yang, H., and Xu, Z.: Relationship between soil erodibility and modeled infiltration rate in different soils, J. Hydrol., 528, 408-418, https://doi.org/10.1016/j.jhydrol.2015.06.044, 2015a.
Wang, L., Zhong, C., Gao, P., Xi, W., and Zhang, S.: Soil Infiltration Characteristics in Agroforestry Systems and Their Relationships with the Temporal Distribution of Rainfall on the Loess Plateau in China, PLoS One, 10, e0124767, https://doi.org/10.1371/journal.pone.0124767, 2015 b.

Wang, T., Xu, H.-1., and Bao, W.-m.: Application of isotopic information for estimating parameters in Philip infiltration model, Water Science and Engineering, 9, 287-292, https://doi.org/10.1016/j.wse.2017.01.005, 2016.

Waterloo, M. J., Schellekens, J., Bruijnzeel, L. A., and Rawaqa, T. T.: Changes in catchment runoff after harvesting and burning of a Pinus caribaea plantation in Viti Levu, Fiji, Forest Ecol. Manag., 251, 31-44, https://doi.org/10.1016/j.foreco.2007.06.050, 2007.

Weynants, M., Montanarella, L., Tóth, G., Arnoldussen, A., Anaya Romero, M., Bilas, G., Borresen, T., Cornelis, W., Daroussin, J, Gonçalves, M., Haugen, L., Hennings, V., Houskova, B., Iovino, M., Javaux, M., Keay, C. A., Kätterer, T., Kvaerno, Si., Laktinova, T., Lamorski, K., Lilly, A., Makó, A., Matula, S., Morari, F., Nemes, A., Patyka, N. V., Romano, N., Schindler, U., Shein, E., Slawinski, C., Strauss, P., Tóth, B., and Wösten, H.: European HYdropedological Data Inventory (EU-HYDI), Publications Office of the European Union, Luxembourg, EUR - Scientific and Technical Research series, 168 pp., https://doi.org/10.2788/5936, 2013.

White, I. and Sully, M. J.: Macroscopic and microscopic capillary length and time scales from infiltration, Water Resour. Res., 23, 1514-1522, https://doi.org/10.1029/WR023i008p01514, 1987.

Wu, G.-L., Yang, Z., Cui, Z., Liu, Y., Fang, N.-F., and Shi, Z.-H.: Mixed artificial grasslands with more roots improved mine soil infiltration capacity, J. Hydrol., 535, 54-60, https://doi.org/10.1016/j.jhydrol.2016.01.059, 2016.

Yang, J.-L. and Zhang, G.-L.: Water infiltration in urban soils and its effects on the quantity and quality of runoff, J. Soil. Sediment., 11, 751-761, https://doi.org/10.1007/s11368-011-0356-1, 2011.

Yilmaz, D., Lassabatere, L., Angulo-Jaramillo, R., Deneele, D., and Legret, M.: Hydrodynamic characterization of basic oxygen furnace slag through an adapted BEST method, Vadose Zone J., 9, 107-116, 2010, https://doi.org/10.2136/vzj2009.0039, 2010.

Zhang, J., Jiao, J., and Yang, J.: In situ rainfall infiltration studies at a hillside in Hubei Province, China, Eng. Geol., 57, 31-38, https://doi.org/10.1016/S0013-7952(99)00126-X, 2000.

Zhang, Y. and Schaap, M. G.: Weighted recalibration of the Rosetta pedotransfer model with improved estimates of hydraulic parameter distributions and summary statistics (Rosetta3), J. Hydrol., 547, 39-53, https://doi.org/10.1016/j.jhydrol.2017.01.004, 2017.

Zhang, Z., Lin, L., Wang, Y., and Peng, X.: Temporal change in soil macropores measured using tension infiltrometer under different land uses and slope positions in subtropical China, J. Soil. Sediment., 16, 854-863, https://doi.org/10.1007/s11368-015-1295-z, 2015.

Zhao, H., Zeng, Y., Lv, S., and Su, Z.: Analysis of soil hydraulic and thermal properties for land surface modeling over the Tibetan Plateau, Earth Syst. Sci. Data, 10, 1031-1061, https://doi.org/10.5194/essd-10-1031-2018, 2018.

Zhao, H. H., Zeng, Y. Y., and Su, Z. B.: Soil Hydraulic and Thermal Properties for Land Surface Modelling over the Tibetan Plateau, Dataset, https://doi.org/10.4121/uuid:61db65b1b2aa-4ada-b41e-61ef70e57e4a, 2017. 
Zhao, Y., Peth, S., Hallett, P., Wang, X., Giese, M., Gao, Y., and Horn, R.: Factors controlling the spatial patterns of soil moisture in a grazed semi-arid steppe investigated by multivariate geostatistics, Ecohydrology, 4, 36-48, https://doi.org/10.1002/eco.121, 2011. 